\title{
Experimental study of flow structure around two in-line bridge piers
}

1 Alireza Keshavarzi MSc, PhD

Professor, Center for Infrastructure Engineering, Western Sydney University, Penrith, NSW, Australia; School of Civil and Environmenta Engineering, University of Technology Sydney, Sydney, NSW, Australia (Orcid: 0000-0003-4388-024X)

2 Chij Kumar Shrestha MSc, PhD

Graduate Student, School of Civil and Environmental Engineering, University of Technology Sydney, Sydney, NSW, Australia

3 Mohsen Ranjbar Zahedani MSc

PhD Student, School of Civil and Environmental Engineering,

University of Technology Sydney, Sydney, NSW, Australia

(corresponding author: mohsen.ranjbar80@gmail.com)
4 James Ball MSc, PhD

Associate Professor, School of Civil and Environmental Engineering, University of Technology Sydney, Sydney, NSW, Australia

5 Hadi Khabbaz MSc, PhD

Associate Professor, School of Civil and Environmental Engineering, University of Technology Sydney, Sydney, NSW, Australia
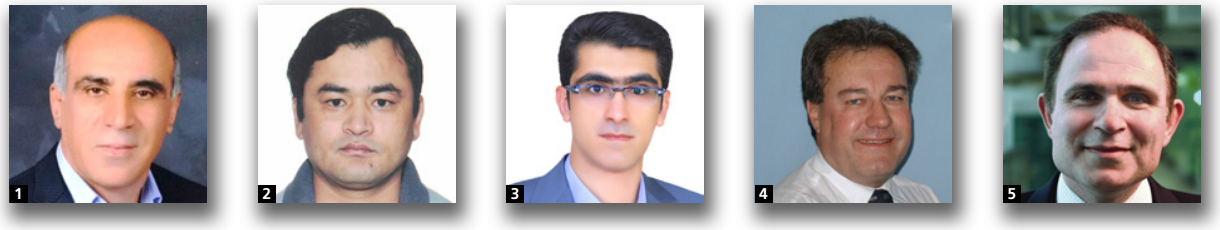

Previous investigations indicate that local scouring is one of the most common causes of waterway bridge failure. The scour mechanism around bridge piers is complicated by the interaction of flow and structure. To explore the local scouring process, it is therefore essential to study the flow-structure interaction around bridge piers. Most previous studies have been based on this interaction around a single pier; however, in practice, many bridges are wide and comprise a number of piers aligned in the flow direction that together support the loading. In this study, a particle image velocimetry technique was used to investigate two-dimensional flow-structure interaction around two in-line bridge piers with different spacings. Various influencing flow characteristics including turbulence intensity, turbulent kinetic energy and Reynolds stresses were calculated in different vertical planes around the bridge piers. Results indicated that the flow characteristics around two in-line bridge piers are very different than for a single pier and the spacing between two in-line piers significantly influences the flow characteristics, particularly in the rear of the piers. Furthermore, for spacing in the range of $2 \leq L / D \leq 3$, stronger turbulence structures occurred behind pier 1 and, as a result, a higher scour depth can be expected around pier 1 .

\section{Notation}

D

$h$

L

Q

$\mathrm{TI}_{u}$

$\mathrm{TI}_{w}$ pier diameter

water depth

centre-to-centre distance between two in-line piers

flow rate

turbulence intensity component in stream-wise direction ( $x$-direction)

turbulence intensity component in vertical

direction ( $z$-direction)

instantaneous velocity component in stream-wise direction ( $x$-direction)

fluctuating component of velocity in stream-wise direction ( $x$-direction)

time-averaged velocity component in stream-wise

direction ( $x$-direction)

mean flow velocity

flume width $\tau_{u v}, \tau_{v w}, \tau_{u w}$ components of Reynolds stress tensor

\section{Introduction}

Complicated flow structures around bridge piers cause local scouring, and represent one of the most important reasons for waterway bridge failure. Many researchers such as Melville (1975), Ettema (1980), Qadar (1981), Chiew (1984), Hamill (1999), Melville and Coleman (2000), Richardson and Davis (2001) and Sheppard (2004) have declared that the basic mechanism of local scour is a system of vortices 
developed around bridge piers. Figure 1 shows different components of flow contributing to the scour around bridge piers.

Melville (1975) reported that a system of vortices (horseshoe vortex and wake vortex), which develops around the bridge pier, is the primary cause of the scour around bridge piers. The horseshoe vortex increases the velocities near the bed, resulting in an increase in the sediment transport capacity of the flow. The wake vortex system keeps the sediment suspended. It also acts as a 'vacuum cleaner', with the bed material carried to the downstream side by the eddies shaded from the pier. According to Qadar (1981), flow in front of a pier separates from the bed and rolls up to form a scouring vortex, which is identified as the basic mechanism of the local scour depth. Chiew (1984) also suggested a concept similar to the conceptual model of Melville (1975). To understand the scour mechanism around a bridge pier, some studies, for example those by Melville and Raudkivi (1977), Ettema (1980), Dargahi (1989), Ahmed and Rajaratanam (1997), Richardson and Panchang (1998), Melville and Coleman (2000), Meneghini et al. (2001), Muzzammil and Gangadhariah (2003), Shrestha et al. (2012), Kumar and Kothyari (2012) and Shrestha et al. (2013), have been conducted on flow structures and scouring around bridge piers. In all the above studies it was concluded that vortices are the most important causes of sediment particle entrainment around bridge piers. The vortices are developed due to flow separation on the bridge pier and may develop a large scour hole downstream from the piers. As reported by Melville and Coleman (2000), the principal features of the flow field at a bridge pier are down-flow at the upstream side of the pier, the horseshoe vortex at the base of the pier, the surface roller at the upstream side of the pier and wake vortices downstream of the pier (see Figure 1).

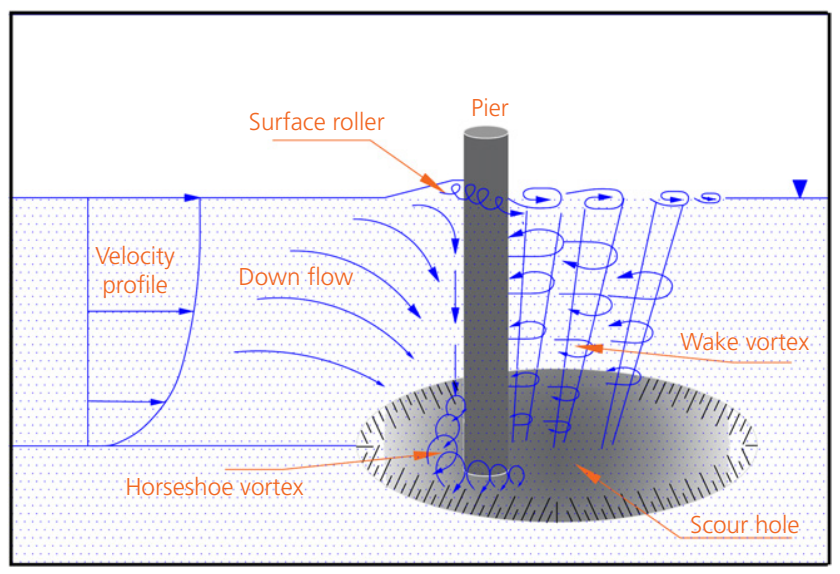

Figure 1. Flow field around bridge piers
Ettema et al. (2011) suggested three categories of pier flow field, depending on ratio of flow depth $(h)$ to pier diameter $(D)$ as follows

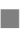
narrow piers $(h / D>1 \cdot 4)$

transitional piers $(0 \cdot 2<h / D<1 \cdot 4)$

wide piers $(h / D<0 \cdot 2)$.

In narrow piers the scour is deepest at the pier face. Ettema et al. (2011) reported that the flow field at narrow piers consists of an interacting and unsteady set of flow features, including: flow impact against the pier face, producing downflow and up-flow with rollers; flow converging, contracting then diverging; the generation, transport and dissipation of a large-scale turbulence structure at the base of the pier foundation junction; detaching shear layer at each pier flank; and wake vortices connected through the pier's wake. For the transition piers there exists almost the same flow field as for narrow piers. However, the flow field (especially the downflow) begins to alter in response to the reduction of the depth of flow or increase in the size of the pier. For wide piers, as the flow approaches the pier, it turns and flows laterally along the pier face before contracting and passing around the side of the pier. A weak down-flow is developed, causing less scouring at the centre of the pier in the upstream side. The wide pier increases flow blockage, which modifies the lateral distribution of approach flow over a longer distance upstream of a pier. Other studies have also been conducted in the field of local scour. For instance, Mohammadpour et al. (2015) studied scour prediction at river bridge abutments over time. In addition, some researchers have used numerical modelling to simulate the flow pattern in different fields of water engineering (e.g. Greifzu et al., 2016; Özkan et al., 2016).

A review of previously published studies indicates that turbulent flow structures around a single bridge pier are complicated. Hence, it is more complicated when two in-line bridge piers obstruct the flow. In this situation, the distance between piers affects the flow structure in addition to other parameters such as the geometry of piers and hydraulics of flow. Additionally, most of the previous studies - for example, by Igarashi (1981), Okajima et al. (2007), Sumner (2010) and Elhimer et al. (2016) - on two in-line columns have been done in wind tunnels for the purpose of aeronautical engineering; however, research on bed scouring around two bridge piers in a tandem arrangement in a river and its linkage to flow structure remains inadequate. Therefore, one of the main objectives of the present research is to analyse the influence of the pier spacing between two in-line circular piers on flow structure and turbulence characteristics. To meet this objective, detailed experimental studies on two-dimensional flow structures around a single pier and two in-line piers with different spacings were undertaken. Particle image velocimetry (PIV) techniques were employed to measure the two-dimensional 


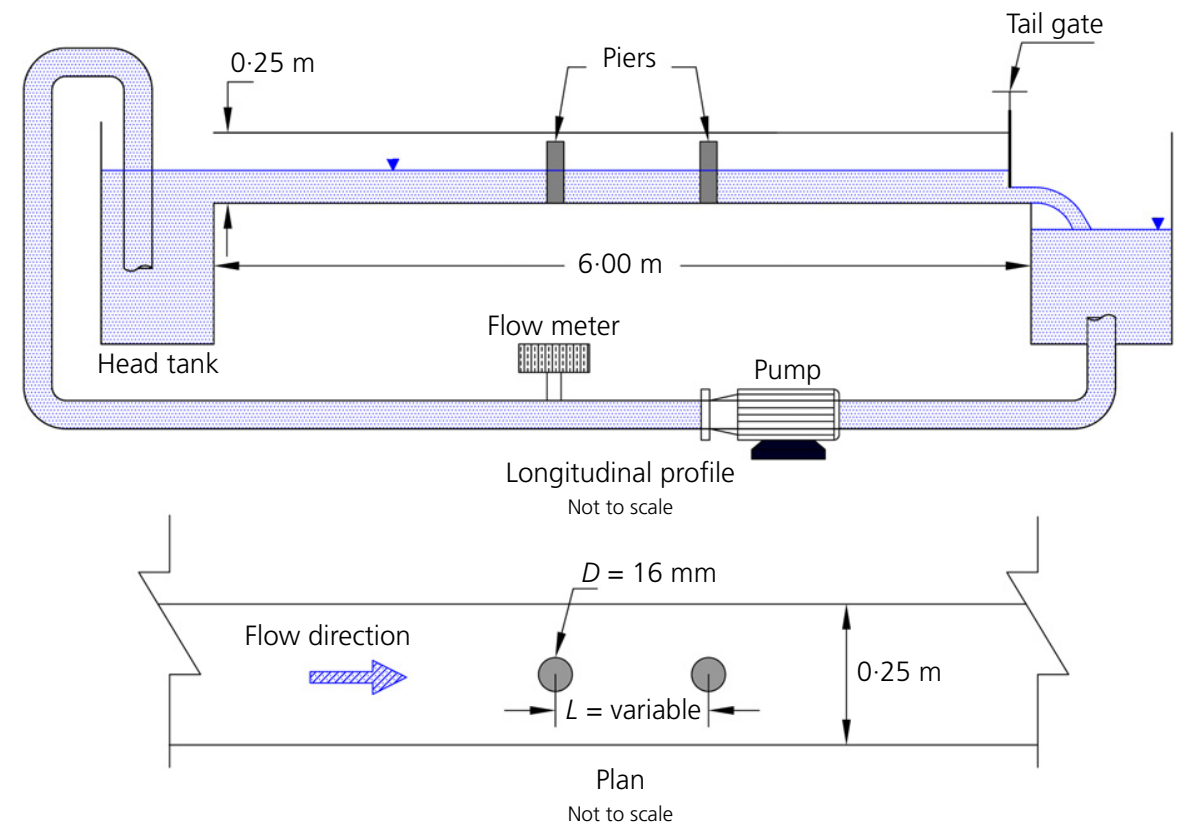

Figure 2. Schematic diagram of the laboratory flume

instantaneous velocity components in vertical planes under no scouring and clear water flow.

\section{Experimental set-up and procedure}

A rectangular flume with nominal dimensions of $6 \mathrm{~m}$ long, $0.25 \mathrm{~m}$ wide and $0.25 \mathrm{~m}$ deep (see Figure 2) was used to study the flow structures around bridge piers under a fixed bed condition using PIV. The flume had a poly(methyl methacrylate) (PMMA) sidewall to enable visualisation of flow conditions during the experimental tests. This flume was equipped with a flow meter, a regulating gate and a pump. Flow discharge was measured using an electromagnetic flow meter (MAG 5000) with an accuracy of $0.4 \%$.

A set of eight experiments (see Table 1) was undertaken with a single pier and two in-line circular piers with different spacing between them $(L / D=1,2,2 \cdot 5,3,4,5$ and 6 , where $L$ was the centre-to-centre distance between two in-line piers and $D$ was the pier diameter). The piers were installed on the centre-line

Table 1. Experimental set-up

\begin{tabular}{|c|c|c|c|c|c|}
\hline Test & $D: \mathrm{mm}$ & $L / D$ & $Q(L / S)$ & $h: \mathrm{mm}$ & $V: \mathrm{m} / \mathrm{s}$ \\
\hline 1 & 16 & Single pier & 3 & 115 & 0.1 \\
\hline 2 & 16 & 1 & 3 & 115 & $0 \cdot 1$ \\
\hline 3 & 16 & 2 & 3 & 115 & $0 \cdot 1$ \\
\hline 4 & 16 & $2 \cdot 5$ & 3 & 115 & $0 \cdot 1$ \\
\hline 5 & 16 & 3 & 3 & 115 & $0 \cdot 1$ \\
\hline 6 & 16 & 4 & 3 & 115 & $0 \cdot 1$ \\
\hline 7 & 16 & 5 & 3 & 115 & $0 \cdot 1$ \\
\hline 8 & 16 & 6 & 3 & 115 & $0 \cdot 1$ \\
\hline
\end{tabular}

of the flume and the upstream pier was located at a distance of $2 \mathrm{~m}$ from the inlet section of the flume. Although all experiments were conducted in a fixed-bed flume with no sediment layer, the geometric and hydraulic parameters were chosen regarding the criteria of local scour around the bridge piers. The pier diameter was carefully chosen so that there was no contraction effect on the depth of scour. According to Melville and Coleman (2000), to avoid the contraction effect, the flume width should be at least ten times greater than the pier diameter. In this study, piers with diameters of $16 \mathrm{~mm}$ were taken for the tests. The flume width to the pier diameter ratio $(W / D)$ was $15 \cdot 62$, satisfying the boundary condition criterion recommended by Melville and Coleman (2000). As classified by Melville and Coleman (2000), for a narrow condition $(D / h<0 \cdot 7)$ flow depth does not affect the local scour depth. Therefore, flow depth was set to $115 \mathrm{~mm}$ and $D / h$ was equal to $0 \cdot 14$, which satisfies the aforementioned criterion of the narrow condition. In all tests the flow rate of $31 / \mathrm{s}$ was supplied to the flume and the mean flow velocity $(V)$ was equal to $0 \cdot 1 \mathrm{~m} / \mathrm{s}$.

PIV was used for the study of flow structure around two in-line bridge piers. In this method, a number of images were recorded to measure the displacement of particles moving within a narrow light sheet. In PIV, the fluid is seeded with small tracer particles, enabling visibility of the fluid motion. The instantaneous displacement of these seeded particles is used to retrieve information on the flow velocity field. An illustration of the PIV system and its components is provided in Figure 3. In order to collect more accurate data, the seeded particles must be able to match the properties of the fluid used for the 


\section{Offprint provided courtesy of www.icevirtuallibrary.com Author copy for personal use, not for distribution}

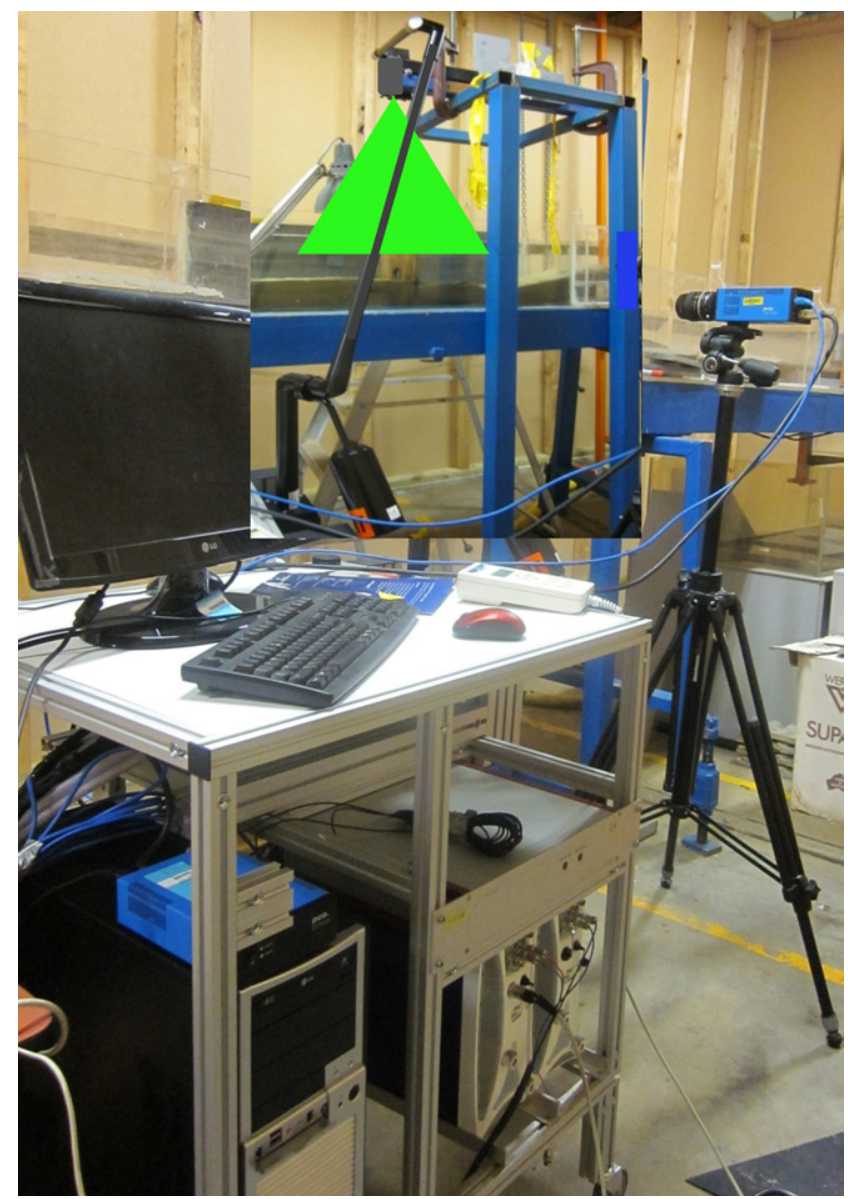

Figure 3. Illustration of PIV system

investigation. The ideal seeding particles have the same density as the fluid being used and should be spherical. Additionally the size of the particles should be small enough so that the time to respond to the particle motion of the fluid is reasonably short and accurately follows the flow. In this study, polyamide 2070 was used for the seeding particles, which were spherical in shape with an approximate mean diameter of $5 \mu \mathrm{m}$ and approximate mean density of $1.016 \mathrm{~g} / \mathrm{cm}^{3}$.

Once the set-up was complete, the seeding particles (polyamide 2070) were added to the flow. These seeding particles were illuminated in the plane of flow at least twice by means of a laser within a short time interval. The light scattered by seeded particles was acquired by a high-resolution digital camera. The displacement of seeding particles between two consecutive images determines the fluid velocity. To extract displacement information from the PIV recordings, the images were processed using a software package called VidPIV, version 4.6 (VidPIV, 2004). All experiments were conducted in the hydraulics laboratory at the University of Technology Sydney (UTS), Australia.

\section{Results and discussion}

Flow structures and the turbulence characteristics of the flow around tandem bridge piers in different vertical planes were studied using the PIV method. PIV measurements were taken at different vertical planes positioned at $Y / D=0,1 \cdot 25,2.5$, $3 \cdot 75$ and $5 \cdot 0$, as shown in Figure 4 . For each plane, the experiments were carried out with a single pier as well as two in-line circular piers with $L / D=1,2,2 \cdot 5,3,4,5$ and 6 . The longitudinal $(u)$ and vertical $(w)$ instantaneous velocity components were determined from analysis of the PIV images collected during individual experiments.

For each plane of measurement, the data along different vertical lines perpendicular to the flow direction have been extracted. The positions and the notations of extraction lines are shown in Figure 5. At the upstream (US) of pier 1, US1 and US2 represent the positions of the extraction line, which are at the distance of $D$ and $2 D$, respectively, from the

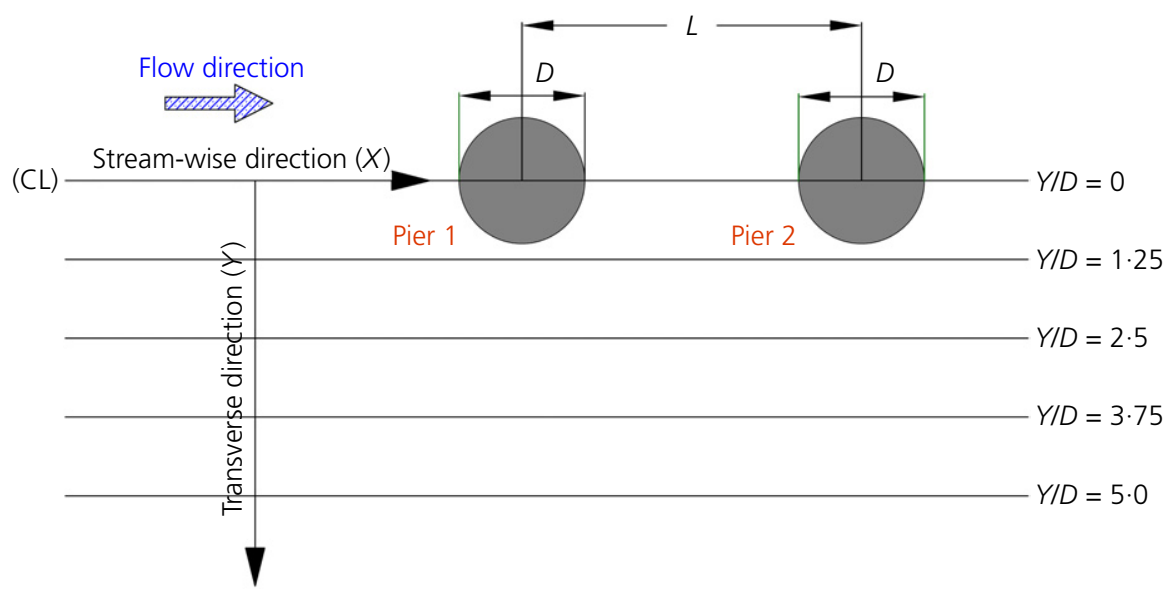

Figure 4. Different axes of PIV measurements (plan view) 


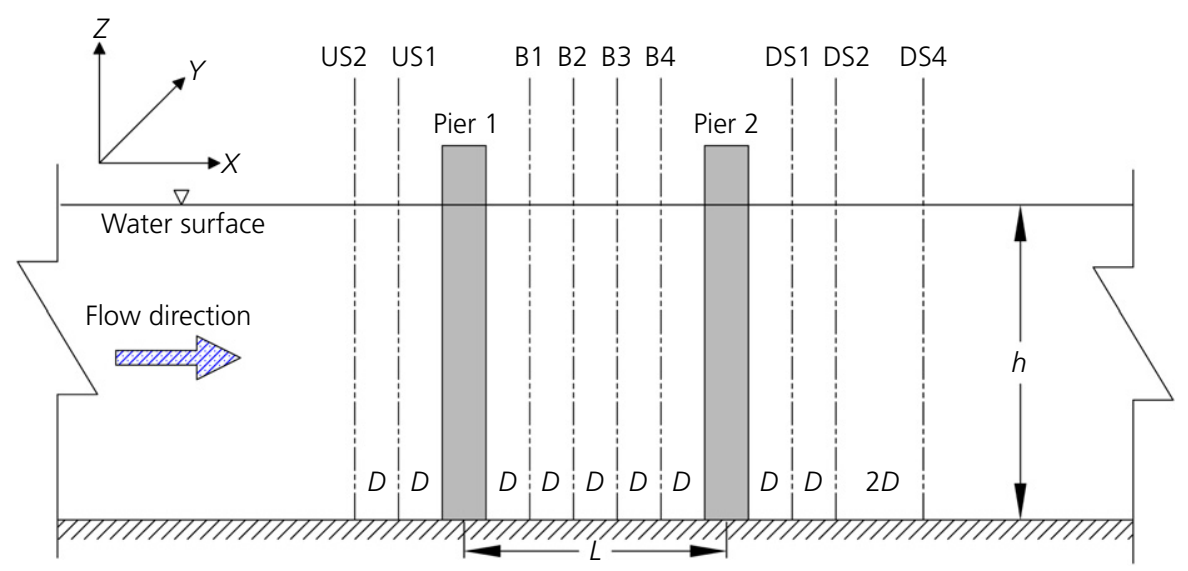

Figure 5. Schematic diagram of different axes of data analysis at upstream and downstream side of the piers in vertical planes (US, B and DS stand for upstream side, between and downstream side of the piers, respectively)

upstream face of pier 1. Similarly, DS1, DS2 and DS4 represent the position of the extraction line located at a distance of $D, 2 D$ and $4 D$, respectively, from the downstream (DS) face of pier 2. In the case of extraction lines between two in-line piers, a typical case of pier arrangement of $L / D=6$ is shown in Figure 5 and is represented by B1, B2, B3 and B4 at a distance of $D$ from each other. However, the numbers of extraction lines between two piers depend upon the distance between them. In this paper, only the figures associated with the cases of a single pier and two in-line piers with spacing $3 D(L / D=3)$ along two vertical planes at $Y / D=0$ and 1.25 are shown as the representative findings.

\subsection{Data analysis}

Flows in most hydraulic structures such as spillways, weirs, irrigation channels and water supply systems are turbulent. In turbulent flow, the velocity and pressure vary with time and space. If $u, v$ and $w$ are the instantaneous velocity components at a point; $\bar{u}, \bar{v}$ and $\bar{w}$ are the time-averaged velocity components; and $u^{\prime}, v^{\prime}$ and $w^{\prime}$ are the fluctuating components of velocity in the stream-wise, transverse and vertical directions, respectively, then the relating equations can be written as

1. $u^{\prime}=u-\bar{u}, \quad v^{\prime}=v-\bar{v}, \quad w^{\prime}=w-\bar{w}$

Based on the velocity fluctuation components, the turbulence intensity (TI) and the turbulent kinetic energy (TKE) of the flow can be determined by Equations 2 and 3, respectively.

2. $\mathrm{TI}=\sqrt{\frac{1}{3}\left(\overline{u^{\prime 2}}+\overline{v^{\prime 2}}+\overline{w^{\prime 2}}\right)}$

3. $\mathrm{TKE}=\frac{1}{2}\left(\overline{u^{\prime 2}}+\overline{v^{\prime 2}}+\overline{w^{\prime 2}}\right)$
Furthermore, the fluctuating parts of the velocity can be used to quantify the Reynolds stress, which is the total stress tensor in a fluid. The components of the Reynolds stress tensor are generally defined as

4. $\quad \tau_{u v}=-\rho \overline{u^{\prime} v^{\prime}}, \quad \tau_{v w}=-\rho \overline{v^{\prime} w^{\prime}}, \quad \tau_{u w}=-\rho \overline{u^{\prime} w^{\prime}}$

\subsection{Flow pattern}

The flow patterns around the bridge piers in a vertical plane are shown in Figure 6. The magnitude of the velocity vector is given by $\left(u^{2}+w^{2}\right)^{1 / 2}$. Additionally, the flow direction in the measurement plane is represented by the inclination of the velocity vectors. Figure 6(a) shows that when the flow is obstructed by a pier, the flow starts to separate from its original direction. Considering the plane at $Y / D=0$, there is generation of a downward flow due to the pressure gradient induced at the upstream face of the bridge pier, as the flow approaches the bridge pier. This downward flow forms a clockwise vortex at the base of the bridge pier. At the immediate downstream side, reversal as well as upward flow are observed, which can be seen in vector diagrams. The flow at the wake of the pier is observed to be in a random direction, which gives a clear indication of the complexity of the flow structure. Furthermore, it is observed that the flow is separated at around $2 D$ on the downstream side measured from the downstream face of the pier. At this separated region, the intensity of up flow is observed to be at a maximum. For the case of $L / D=1$, there is not any space between the piers. Hence, the wake is formed only behind pier 2 . For $L / D=2$, the existence of the wake is not noticed behind pier 1 . However, most of the velocity vectors between the two piers represent the existence of reverse and upward flows. As the spacing increases to $L / D=3$, flow separation is observed at a distance of around $1.5 \mathrm{D}$ from the downstream face of Pier 1, as is shown in Figures 6(c) and 6(d). Additionally, rotation of the flow is observed at the immediate 


\section{Offprint provided courtesy of www.icevirtuallibrary.com Author copy for personal use, not for distribution}

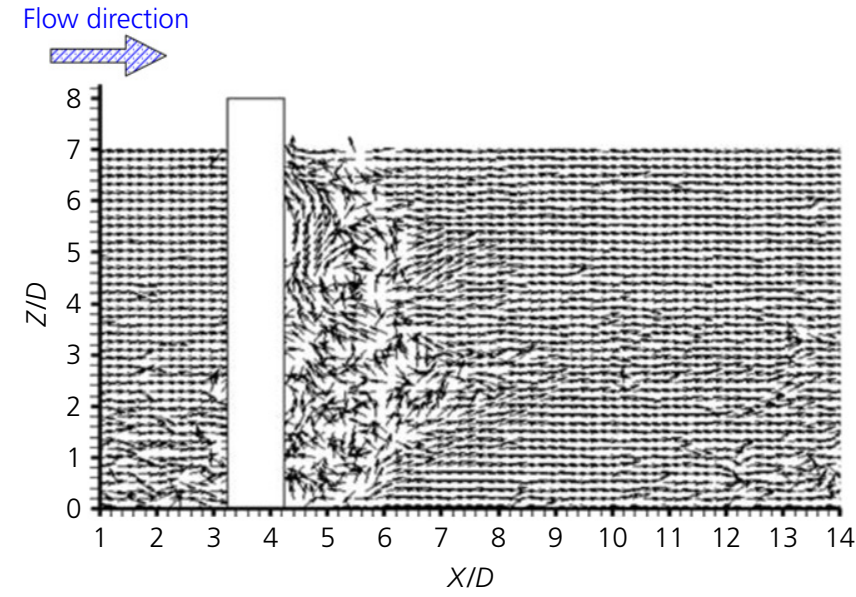

(a)

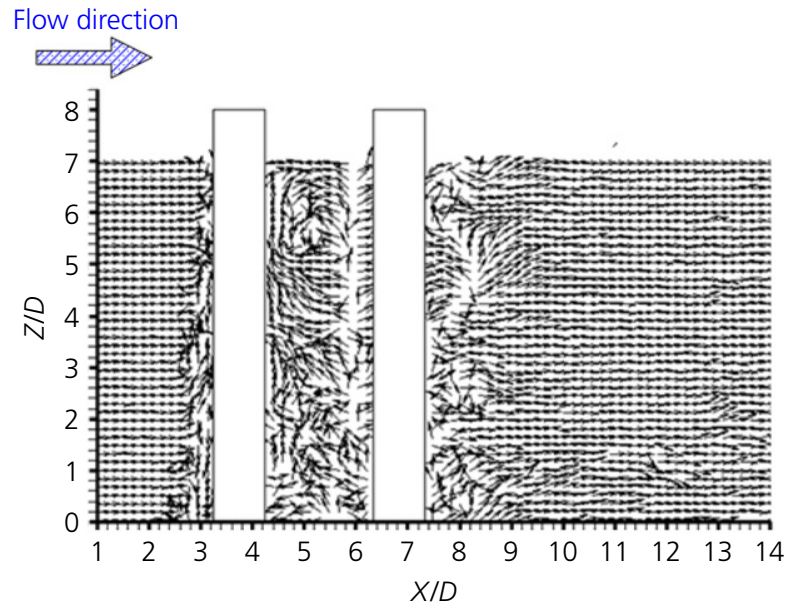

(c)

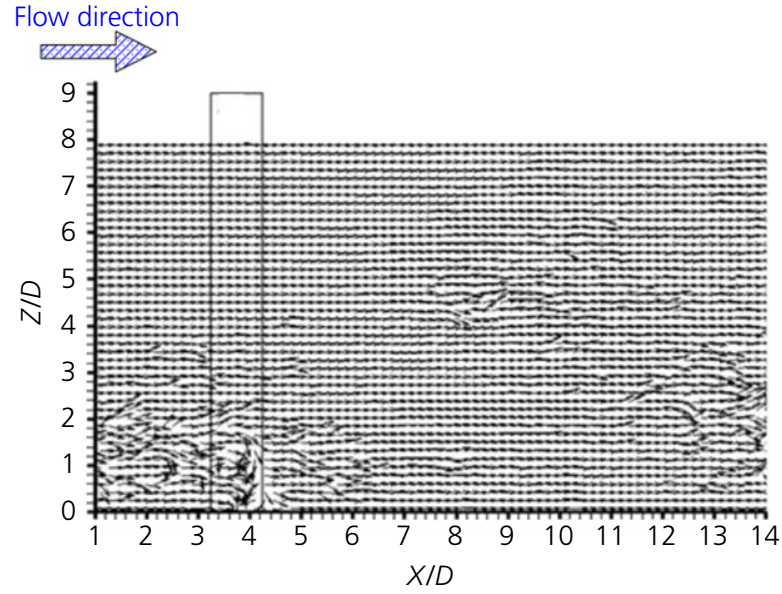

(b)

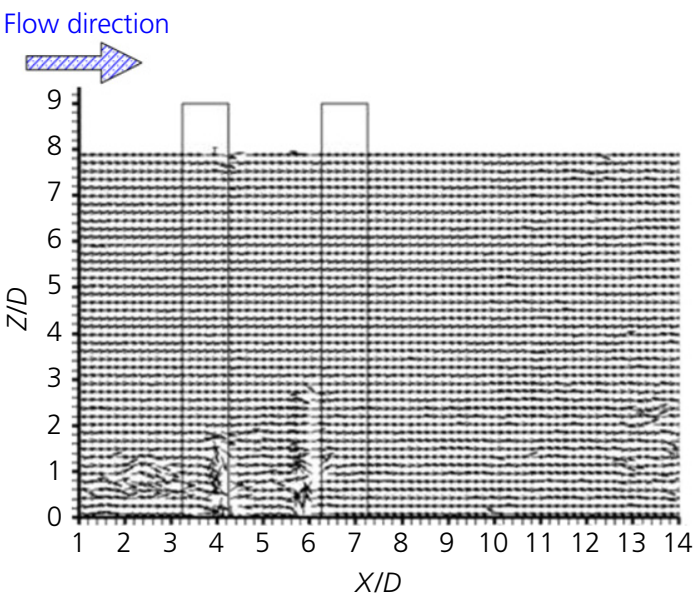

(d)

Figure 6. Flow patterns for (a), (b) single pier and (c), (d) two in-line piers cases

downstream side of pier 1 . On analysing the vector and streamline diagrams for $L / D=4,5$ and 6 , a similar flow pattern is observed; and the flow between two piers is separated at a distance of $2.5 \mathrm{D}$ measured from the downstream face of pier 1 and the rotation of the flow exists. In all cases, the recirculation of the flow exists behind pier 2. The anticlockwise recirculation of the flow, starting from near the bed level close to the pier, diminishes towards the downstream at the middle layer of the depth of flow. For all sets of combinations, the flow patterns at the upstream side of the piers are found to be almost similar, whereas at the downstream side of the pier, the flow pattern is different in various conditions. Comparing the flow behind the single pier and two in-line piers, it is observed that the wake behind the single pier is larger than that of the two in-line piers cases.

\subsection{Time-averaged velocity components}

Figures 7 and 8 show the contour plots and profile plots of the velocity component $(u)$ in a vertical plane for the single pier case. In these figures, the stream-wise velocity component $(u)$ is normalised by the mean flow velocity $(V)$. For the case of a single pier in the plane at $Y / D=0$ the value of $u / V$ at the upstream side of the pier is approximately equal to 1 at a distance $X / D \geq 1.25$ measured from the upstream face of the pier. As the flow approaches the pier, the value of $u / V$ becomes smaller and finally reduces to zero at the face of the pier. At the downstream side of the pier, it is clear from the contour plot that the value of $u / V$ ranged from -0.6 to 0 at the wake close to the downstream face of the pier. The negative value of $u / V$ indicates the presence of the reverse flow in this region. The value of $u / V$ increases as the flow moves further downstream and ultimately comes to the normal flow condition. The contour plot of $u / V$ at the plane $Y / D=1.25$ is shown in Figure 7(b). This figure shows that the maximum value of $u / V$ occurs at the side of the pier, which is approximately equal to $1 \cdot 2$.

The contour plots of $u / V$ for the case of two in-line piers with $L / D=3$ are shown in Figure 9. Similarly, Figures 10 and 11(a) show the velocity profiles of $u / V$ at different positions of the 


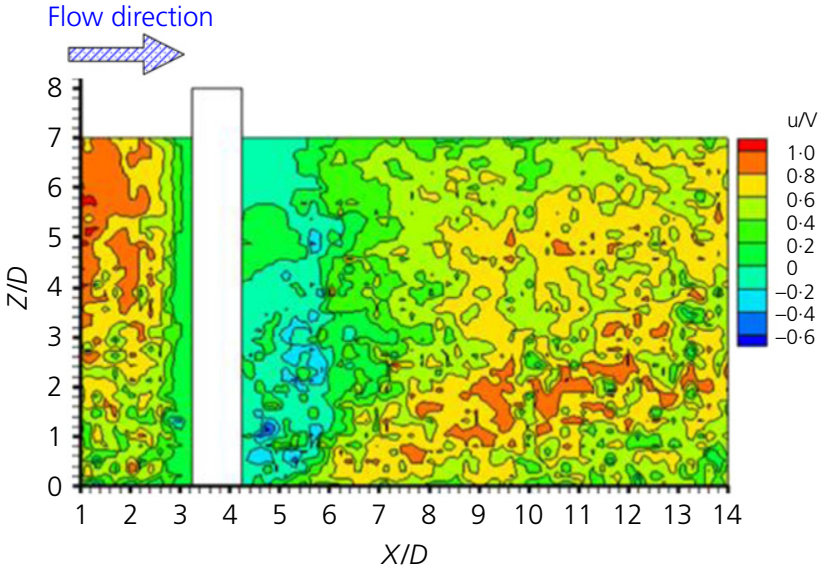

(a)

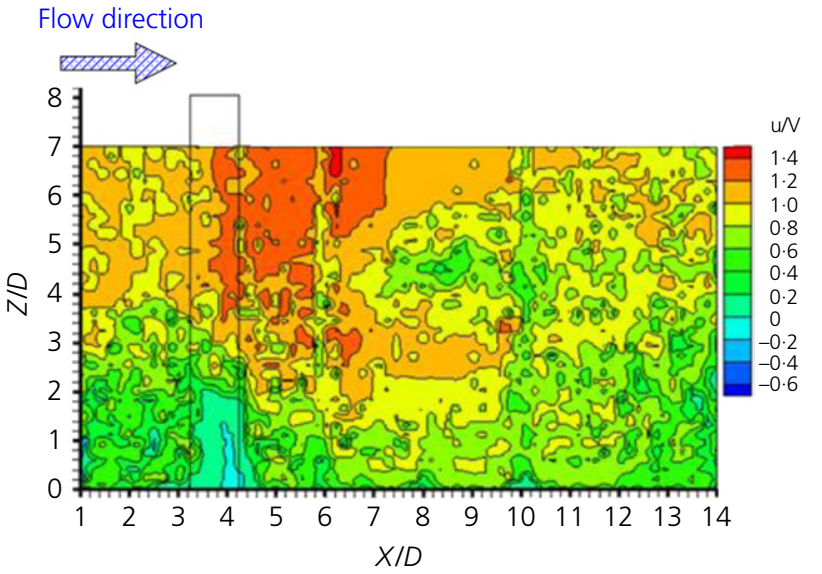

(b)

Figure 7. Contour plots of stream-wise velocity component for the single pier case in different vertical planes: (a) at $Y / D=0$; (b) at $Y / D=1 \cdot 25$
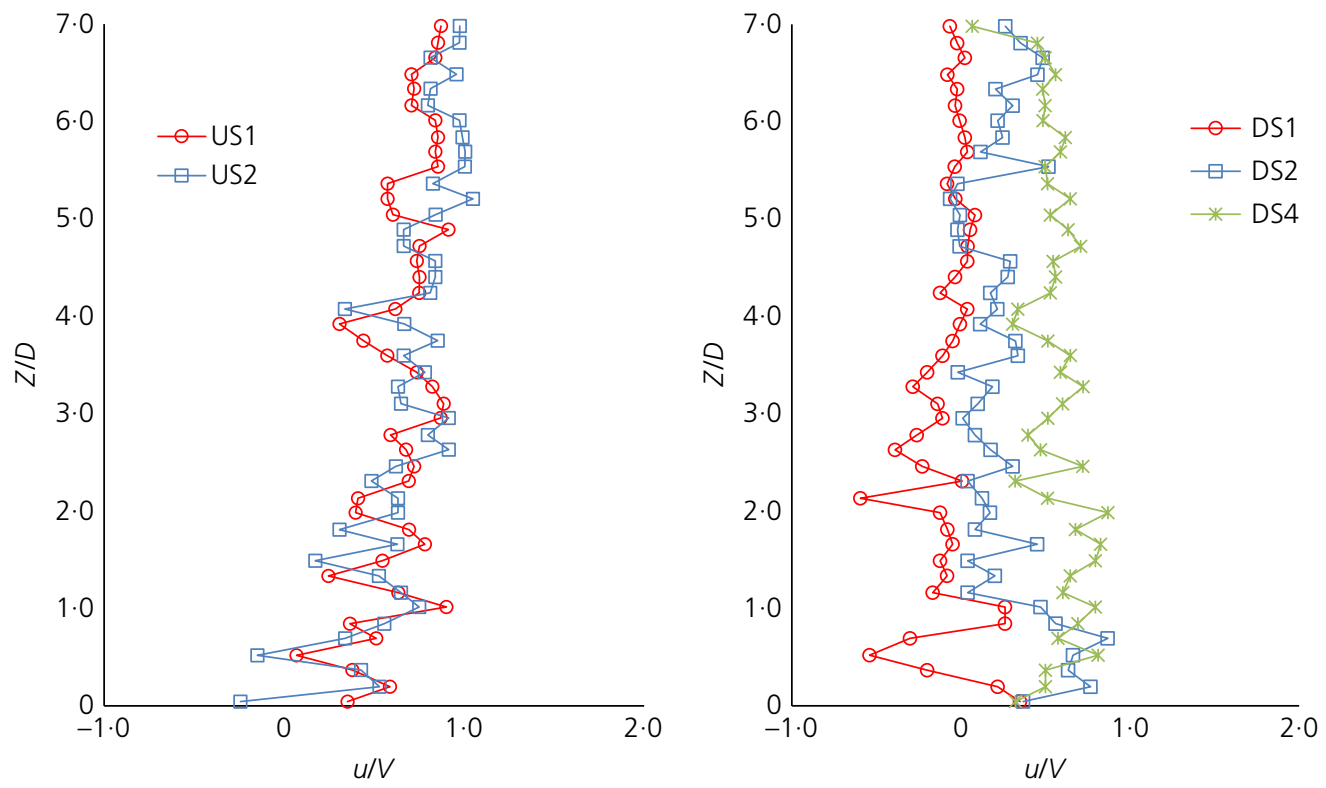

Figure 8. Profile plots of stream-wise velocity component for the single pier case in vertical plane at axis of symmetry: (a) upstream side; (b) downstream side

upstream, downstream sides and between of the piers for $L / D=3$. For the case of two in-line piers, at the upstream side of pier 1 , the distributions of $u / V$ are almost the same for all the pier arrangements. The value of $u / V$ remains 1 when the flow reaches about $1 D$ distance, measured from the upstream face of pier $1 ; u / V$ then decreases gradually as the flow moves towards the pier. This type of distribution is also observed in the single pier case. However, for the downstream side of pier 2 and between the two in-line piers, the distribution of $u / V$ is varied with respect to the spacing between two piers. When $L / D=1$, at the downstream side of pier 2 very close to the pier, the minimum $u / V$ is approximately equal to $-0 \cdot 2$, quite similar to the value obtained for the single pier case. A gradual increase in the value of $u / V$ is noticed with increase in the distance from the downstream face of pier 2. This condition is applied to all of the arrangements of two in-line piers. For the spacing $L / D \geq 2$, the value of $u / V<0 \cdot 5$ is reached within the zone of around $2 D$, measured from the downstream face of pier 2 .

Considering the flow between two in-line piers, the value of $u / V$ for the case of $L / D=2$ varies between $-0 \cdot 5$ and 0 . 


\section{Offprint provided courtesy of www.icevirtuallibrary.com Author copy for personal use, not for distribution}

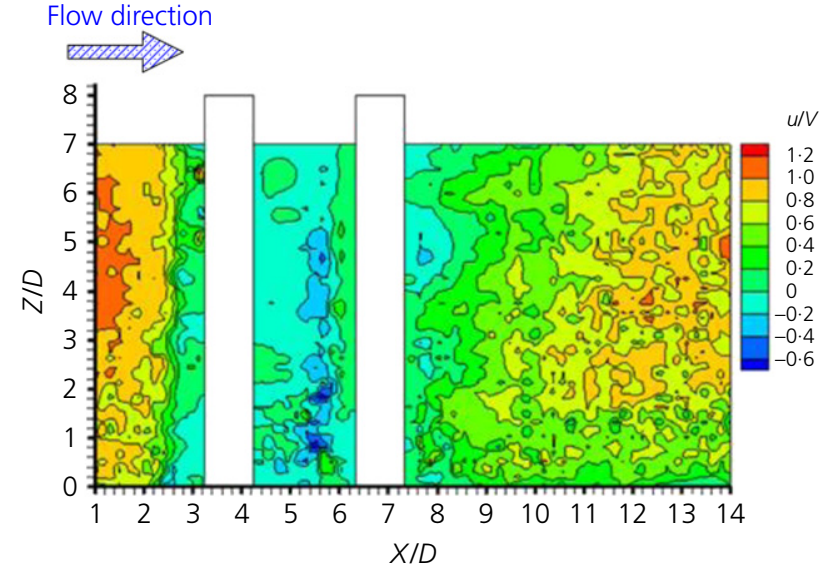

(a)

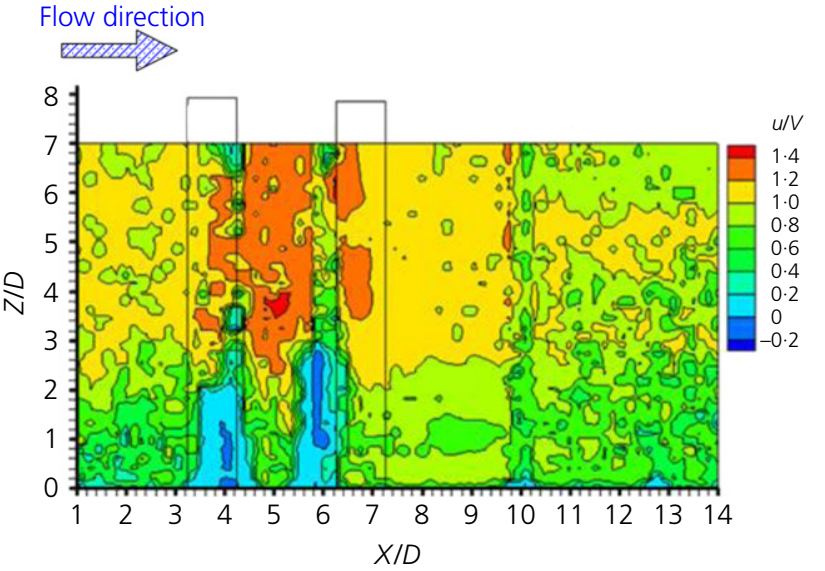

(b)

Figure 9. Contour plots of stream-wise velocity component for the case of two in-line piers with $L / D=3$ in different vertical planes: (a) at $Y / D=0$; (b) at $Y / D=1 \cdot 25$

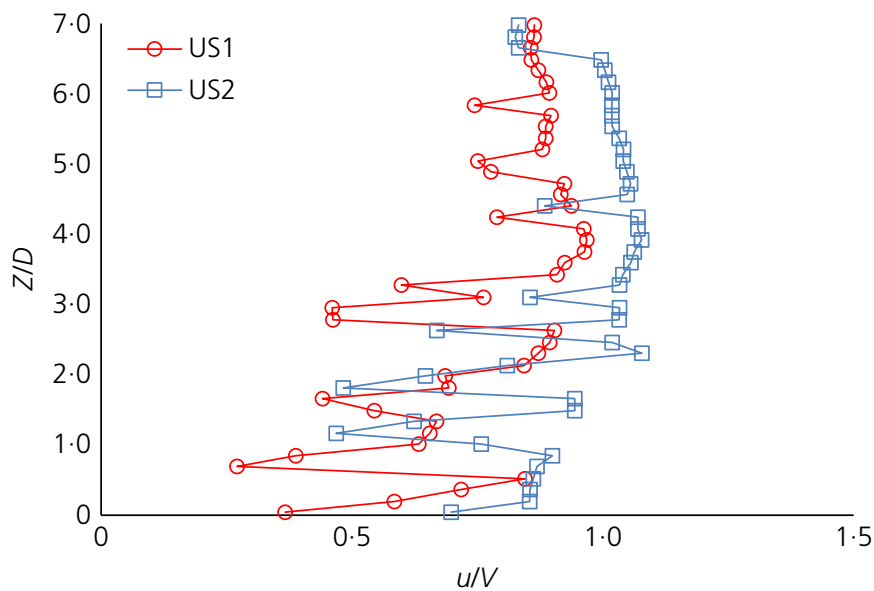

(a)

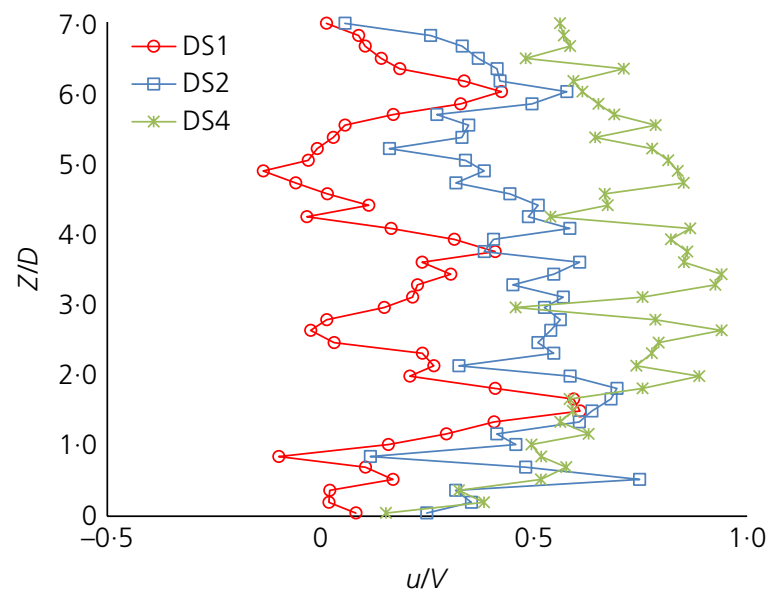

(b)

Figure 10. Profile plots of stream-wise velocity component for two-pier case with $L / D=3$ in vertical plane at axis of symmetry: (a) upstream side; (b) downstream side

The negative value of $u / V$ indicates the reverse flow within the region. As the spacing between the two piers increases, the value of the stream-wise component of the flow velocity approaching pier 2 becomes positive. For $L / D>3$, the maximum value of the stream-wise component of approach flow for pier 2 is $u / V=0 \cdot 4$. However, the contour plots of the plane at $Y / D=1.25$ show that all the arrangements of piers experience the maximum value of $u / V=1.2$ at the side of the piers. In this plane, for $L / D \leq 3$, the maximum value of $u / V$ is at the side of both piers including the gap between the two piers. However, for $L / D \geq 4$, the value of $u / V$ decreases to approximately equal to 1 as the flow approaches pier 2 .

The contour plots for the vertical velocity components $w / V$ are presented in Figure 12 for the single pier in the plane at
$Y / D=0$ and $1 \cdot 25$. In addition, the distribution profiles of $w / V$ are illustrated in Figure 13 at different positions of the upstream and downstream sides of the single pier. At the upstream side of the pier, the range of vertical velocity component $w / V$ is from $-0 \cdot 3$ to $0 \cdot 2$. The negative value represents the down flow. The maximum value of this down flow occurs at the upstream side. As this down flow interacts with the boundary layer, a horseshoe vortex is formed at the base, just upstream of a pier. This horseshoe vortex system interacts with the bed, resulting in more turbulence near the bed. Distribution of $w / V$ at US1 and US2 of the upstream side of the pier is shown in Figure 13(a). This indicates the generation of high turbulence near the bed, which is evident from more fluctuation of $w / V$. At the downstream side of the pier, an upward flow region extending towards the free surface occurs. The maximum value of $w / V$ is 

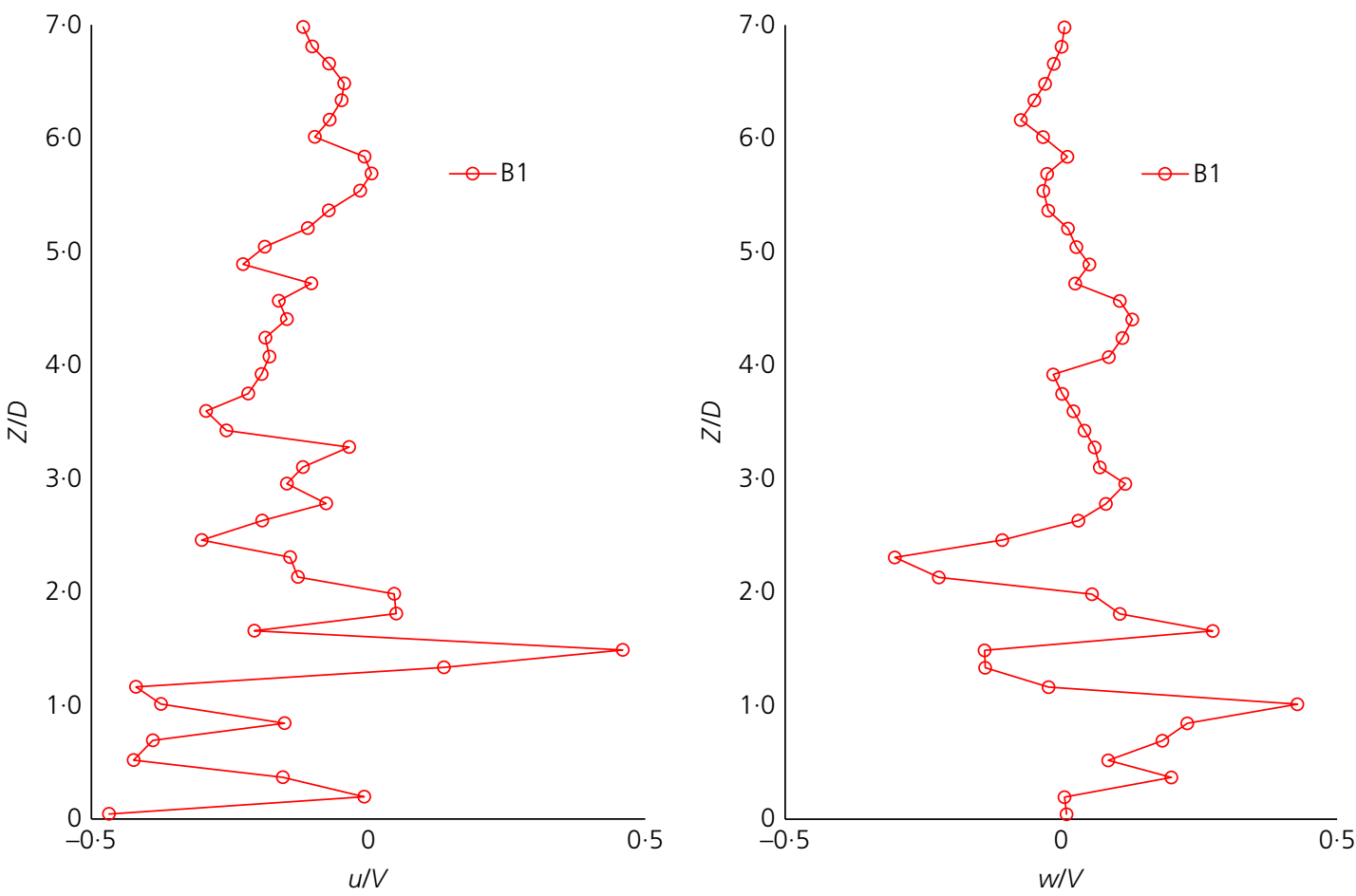

Figure 11. Profile plots of velocity components between two piers with $L / D=3$ in vertical plane at axis of symmetry (a) stream-wise component; (b) vertical component

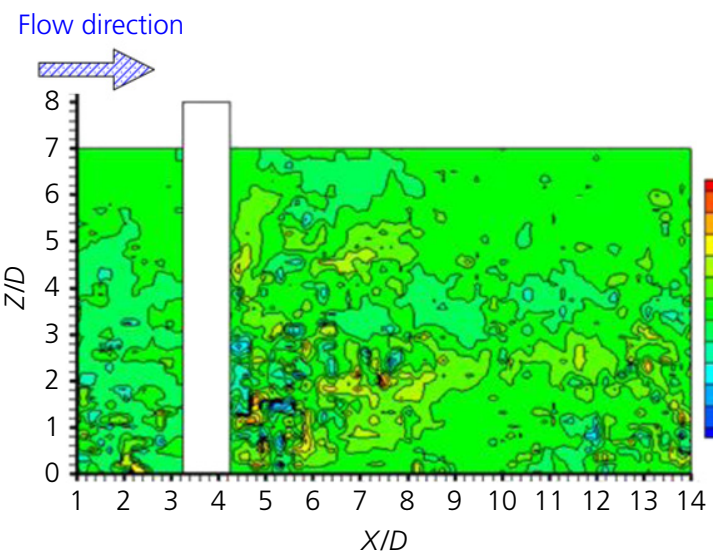

(a)

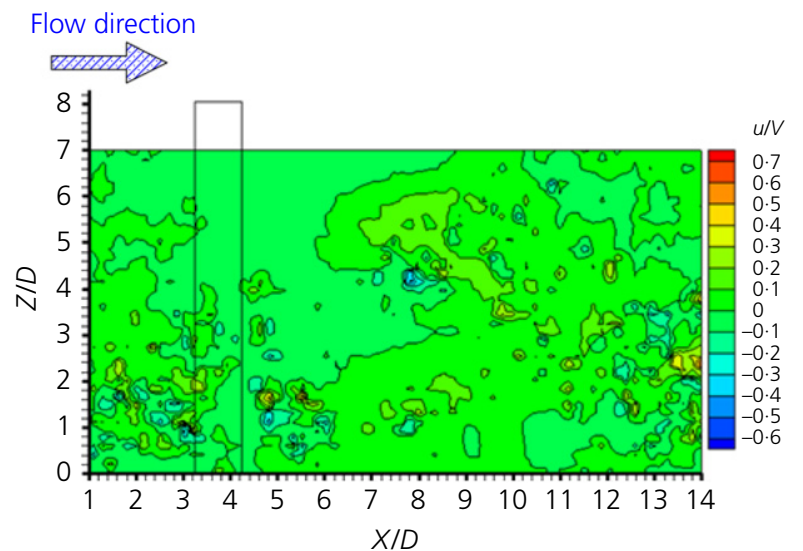

(b)

Figure 12. Contour plots of vertical velocity component for the single pier case in different vertical planes: (a) at $Y / D=0$; (b) at $Y / D=1 \cdot 25$

approximately equal to 0.5 at a distance of about $1.5 \mathrm{D}$ measured from the downstream face of the pier. Furthermore, the maximum and minimum value of $w / V$ occur close to the bed within the wake. Distribution of $w / V$ in different positions DS1, DS2 and DS4 at the downstream side of the pier, as shown in Figure 13(b), shows that the vertical velocity component fluctuates heavily near the bed. Significant fluctuation of $w / V$ is observed up to the distance of $4 D$ measured from the downstream face of a pier. Figure 12(b) shows the contour plot of $w / V$ in the $Y / D=1.25$ plane. It illustrates that down flow of magnitude $0 \cdot 2$ is observed just upstream from a pier and at the side of the pier. At the downstream side of this plane, no significant down flow or up flow is noticed.

In the case of the bridge piers with two in-line piers, at a distance of about $1 D$ measured from the upstream face of pier 1 , the flow is observed as expected. Hence, considering the flow in this region, at the upstream side of pier 1, the down flow is 


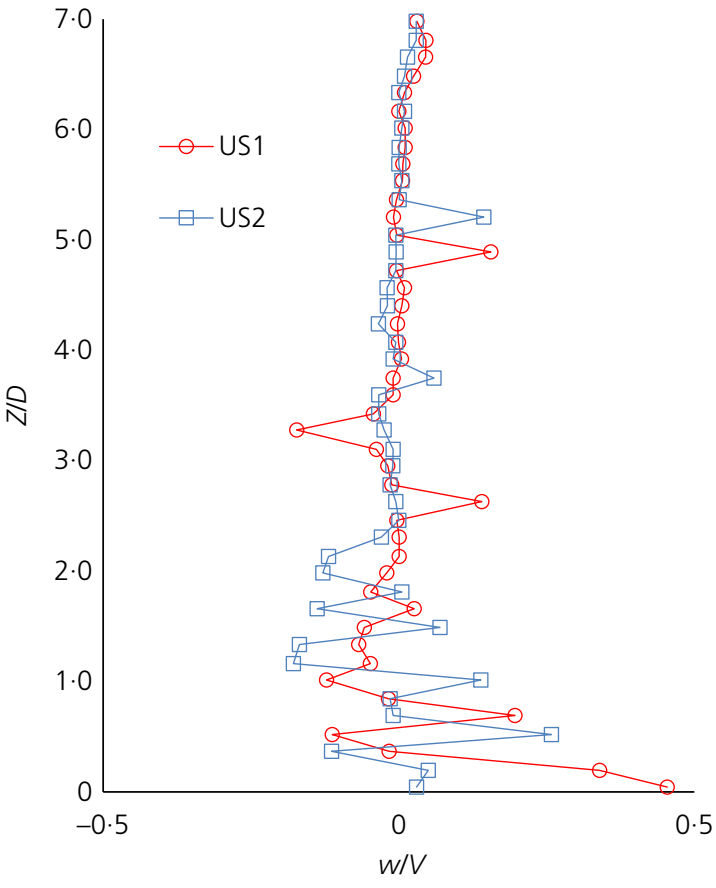

(a)

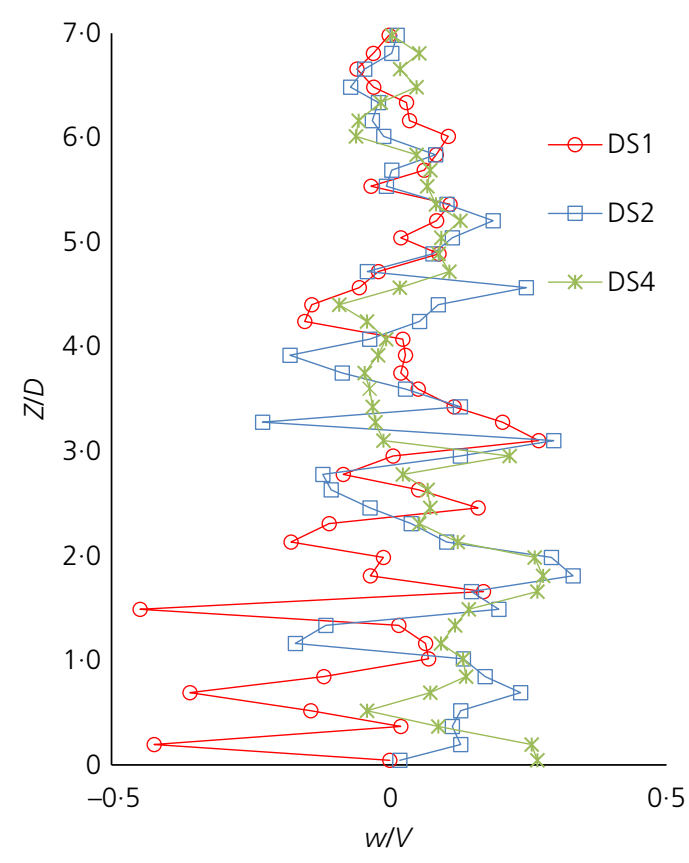

(b)

Figure 13. Profile plots of vertical velocity component for the single pier case in vertical plane at axis of symmetry (a) upstream side; (b) downstream side

observed in all of the cases of two in-line pier arrangements. The maximum value of down flow with $w / V$ approximately equal to $0 \cdot 2$ occurs close to the bed, near the pier base. This is also presented in Figure 14(a). Furthermore, the distribution of $w / V$ at US2, shown in Figure 15(a), indicates no down flow throughout the flow depth. In the plane $Y / D=1 \cdot 25$, as shown in Figure 14(b), down flow with $w / V$ is approximately equal to $0 \cdot 1$, observed at the upstream and at the side of pier 1 . Similar

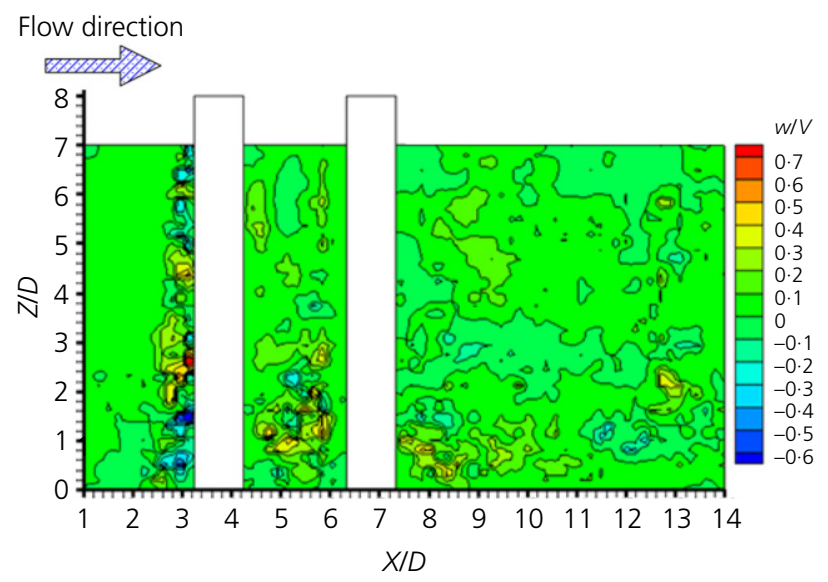

(a) flow is observed at the upstream side of pier 1 for all the values of $L / D$.

Considering the flow between two in-line piers, for $L / D=2$, the gap experiences positive values of $w / V$ ranging from $0 \cdot 1$ to $0 \cdot 3$. This shows the existence of up flow throughout the gap. As no down flow is noticed at the upstream side of pier 2, the horseshoe vortex in this region is not formed. For $L / D=3$,

Figure 14. Contour plots of vertical velocity component for two-pier case with $L / D=3$ in different vertical planes: (a) at $Y / D=0$; (b) at $Y / D=1 \cdot 25$

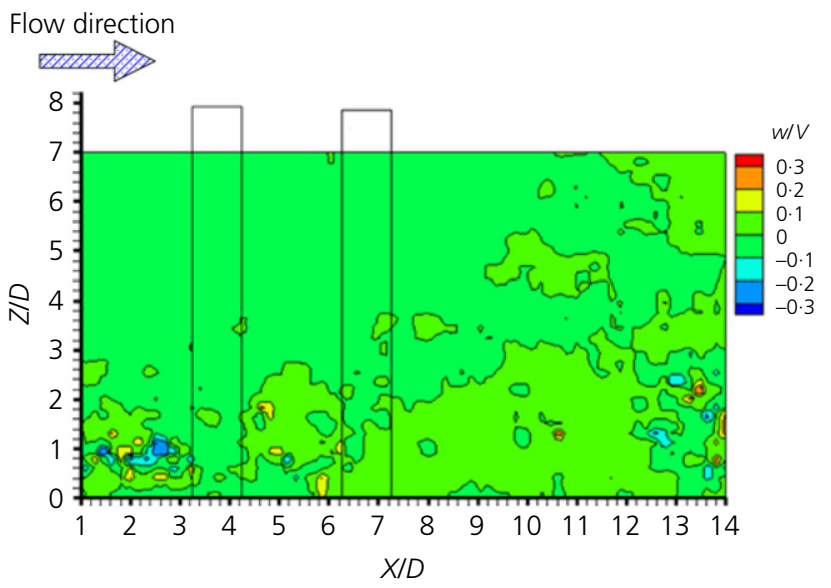

(b) 


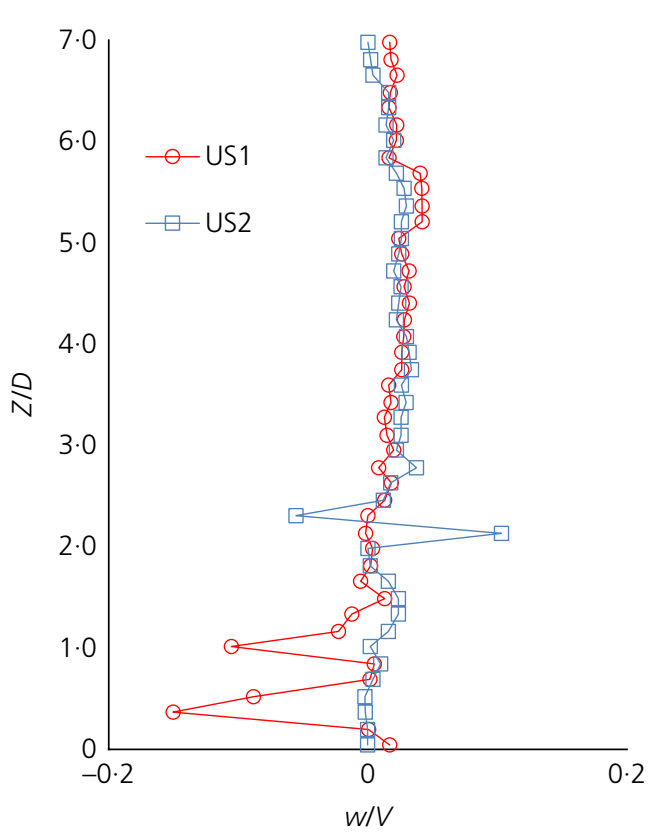

(a)

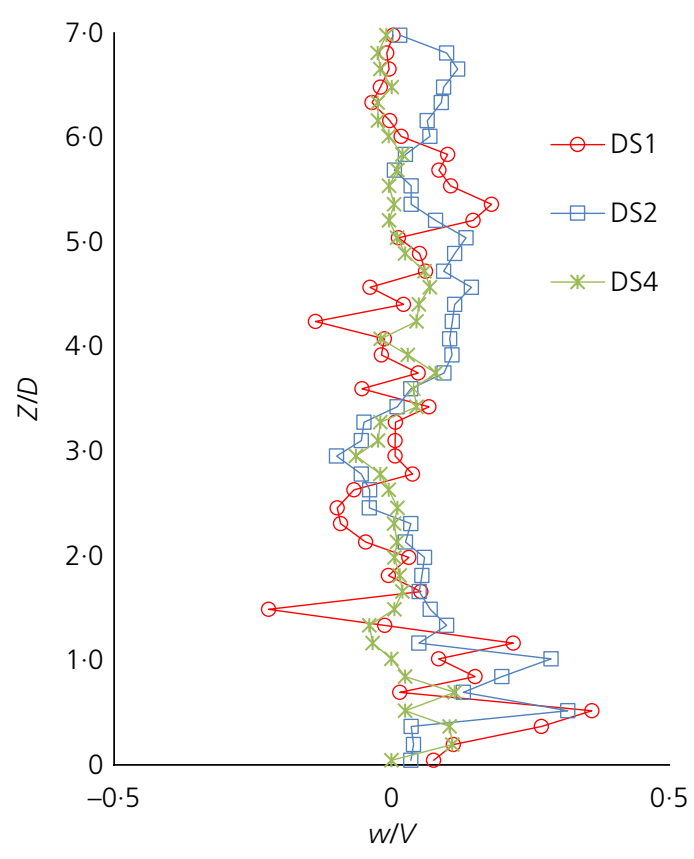

(b)

Figure 15. Profile plots of vertical velocity component for two-pier case with $L / D=3$ in vertical plane at axis of symmetry: (a) upstream side; (b) downstream side

stronger up flow is noticed than that of $L / D=2$. However, the horseshoe vortex at the base of pier 2 is not noticed. For $L / D \geq 4$, the maximum value of $w / V$ is approximately equal to $0 \cdot 5$, which is observed about $2 D$ downstream measured from the face of pier 1 . As the flow approaches pier 2, the intensity of upward flow decreases and eventually down flow is noticed. The horseshoe vortex in front of pier 2 is expected to form near the base of pier 2. However, the size of the horseshoe vortex is smaller than that of pier 1 .

At the downstream side of pier 2, for all pier arrangements, upward flow exists with the maximum value of $w / V=0 \cdot 2$, which is about $50 \%$ smaller than that of the single pier case and about $30 \%$ smaller than the case of pier 1 in the two-pier case. This can be attributed to the sheltering effect of pier 1 . The flow structures at the wake of pier 2 are totally different from the single pier case. For $L / D \leq 4$, a small zone of recirculation forms close to the free surface. The distribution of $w / V$ at DS1, DS2 and DS4 for $L / D \leq 4$ is similar. However, for $L / D>4$ the distribution of $w / V$ at DS1 has a significant negative value close to the bed. The distribution of $w / V$ at DS2 and DS4 is similar in all the cases of two in-line pier arrangements.

According to the values of $u / V$ and $w / V$ for two in-line piers with various spacing, upward and reverse flows form between the two piers for $L / D=2$. Pockets of strong upward and reverse flow occur close to the bed. As the spacing increases to $L / D=3$, stronger upward flow and reversed flow than in the case of $L / D=2$ is identified close to the bed, which is observed at the flow separation zone. As the flow separation for $L / D=3$ occurs close to pier 2, the strength of flow (upward and reverse) at the downstream side close to pier 1 is higher in the case of $L / D=2$ than that in the case of $L / D=3$. When the spacing between two in-line piers increases, the strength of upward flow in the wake of pier 1 decreases. As the flow moves towards pier 2, a gradual increase in stream-wise velocity and a decrease in the vertical velocity component are observed. At the wake of pier 2, a very weak stream-wise velocity component of magnitude $u / V<0.2$ is noticed for all the cases. However, the magnitude of upward flow increases with increase in the spacing between the two piers.

\subsection{Turbulence intensity components}

The root mean square (RMS) values of the components of velocity fluctuations $\left(u^{\prime}\right.$ and $\left.w^{\prime}\right)$ give the turbulence intensity components. In this study, $\mathrm{TI}_{u}$ and $\mathrm{TI}_{w}$ in the stream-wise direction and the vertical direction, respectively, are normalised using the mean flow velocity, $V$.

The contour plots of $\mathrm{TI}_{u} / V$ for the single pier for the planes $Y / D=0$ and 1.25 are shown in Figures 16(a) and 16(b). At the upstream side of a pier in plane $Y / D=0$, the results show that the pocket of higher value of $\mathrm{TI}_{u} / V$ exists close to the bed and decreases with increasing distance towards the free surface. In the case of the plane at $Y / D=1 \cdot 25$, a similar distribution of $\mathrm{TI}_{u} / V$ is noticed at the upstream and side of a pier. At the downstream side of a pier for a plane $Y / D=0$, the maximum value of $\mathrm{TI}_{u} / V=0.8$ is observed close of the pier within the 


\section{Offprint provided courtesy of www.icevirtuallibrary.com Author copy for personal use, not for distribution}

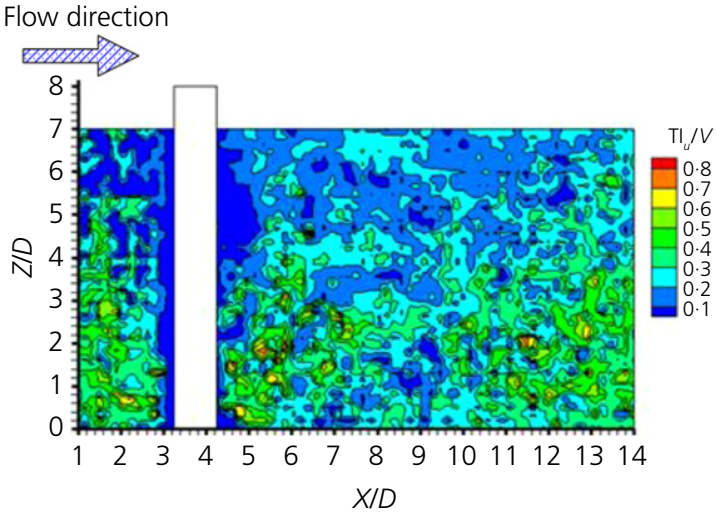

(a)

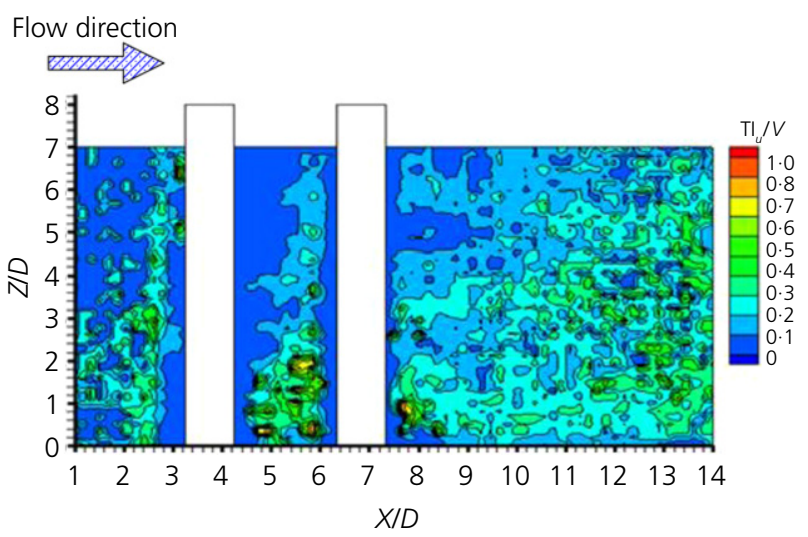

(c)

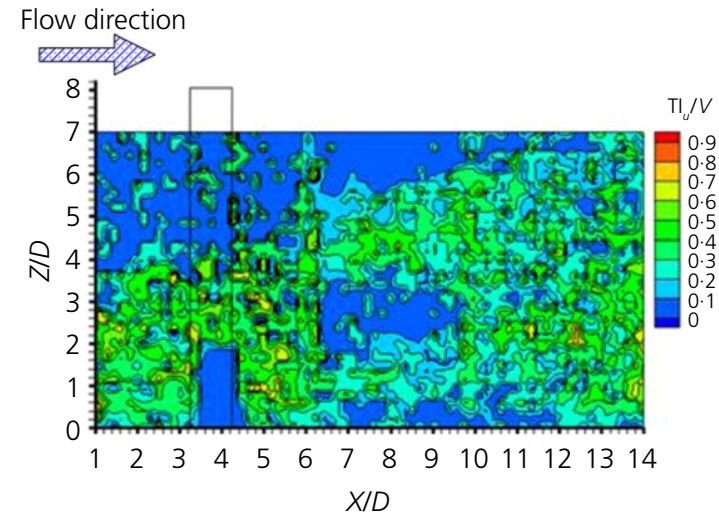

(b)
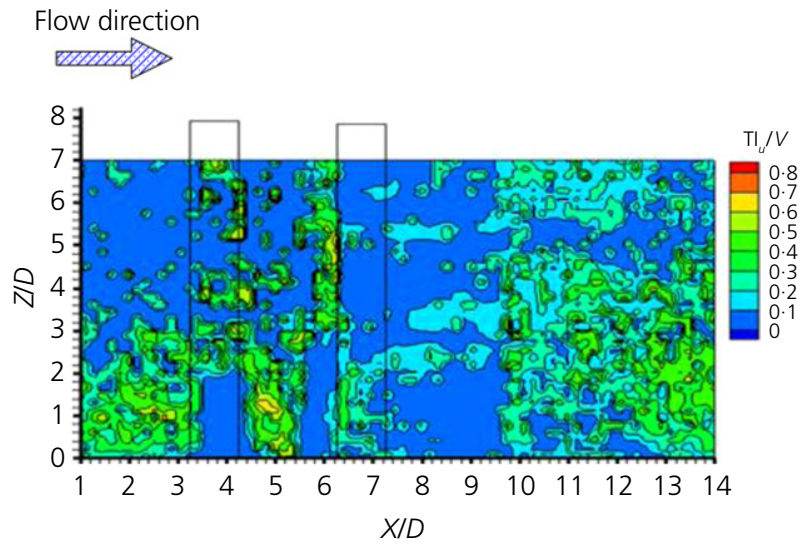

(d)

Figure 16. Contour plots of stream-wise turbulence intensity component for: (a) single pier at $Y / D=0$; (b) single pier at $Y / D=1 \cdot 25$; (c) two piers with $L / D=3$ at $Y / D=0$; (d) two piers with $L / D=3$ at $Y / D=1 \cdot 25$

distance of about $1 \cdot 5 \mathrm{D}$ measured from the downstream face of a pier. The pocket of higher value of $\mathrm{TI}_{u} / V$ is extended to the mid-depth of the flow. For a plane $Y / D=1 \cdot 25$, slightly weaker $\mathrm{TI}_{u} / V$ is noticed at the downstream side. However, the zone of higher value of $\mathrm{TI}_{u} / V$ is observed approximately at the same positions as that in the $Y / D=0$ plane.

For the two in-line pier cases, Figures 16(c) and 16(d) show the contour plots of $\mathrm{TI}_{u} / V$ for $L / D=3$. The results show that, at the upstream side of pier 1 , the maximum value of $\mathrm{TI}_{u} / V=0.5$ is observed in the zone close to the bed for the case of a single pier. Careful analysis of the flow between two piers for $L / D \leq 4$ shows that the higher values of $\mathrm{TI}_{u} / V$ exist close to the bed. It can be noted that the higher value zone is extended throughout the gaps. For $L / D=6$, the maximum value of $\mathrm{TI}_{u} / V$ occurs close to pier 1 at a distance of about $1 \cdot 5 \mathrm{D}$ measured from the face of pier 1 . As the flow approaches pier $2, \mathrm{TI}_{u} / V$ is reduced by about $60 \%$ of the maximum value, observed at the downstream side of pier 1 .

At the downstream side of pier 2, a decrease in the values of $\mathrm{TI}_{u} / V$ is noticed, when compared with the values at the downstream side of pier 1 . The value of $\mathrm{TI}_{u} / V$ at the downstream side of pier 2 increases with increase in the spacing between the two piers. For $L / D \leq 3$ at the plane $Y / D=1.25$ a significant reduction in the value of $\mathrm{TI}_{u} / V$ can be seen at the downstream side of pier 2. However, for $L / D \geq 4$, it is observed that the distribution of $\mathrm{TI}_{u} / V$ at the downstream side of pier 2 is similar to that of the single pier case.

The distribution of the vertical components of normalised turbulence intensity $\mathrm{TI}_{w} / V$ for the single pier case in two different vertical planes at $Y / D=0$ and 1.25 is presented in Figures 17(a) and 17(b). The contour plots at the upstream side of the pier show that the magnitude of $\mathrm{TI}_{w} / V$ is significantly smaller than that of $\mathrm{TI}_{u} / V$. The maximum value of $\mathrm{TI}_{w} / V$ is about 0.2 and occurs close to the bed at US1. Then it decreases with increase of the distance towards the free surface. At the downstream side of the pier, the distribution of $\mathrm{TI}_{w} / V$ is similar to the distribution of $\mathrm{TI}_{u} / V$ with approximately the same value. However, for the contour plot in the plane $Y / D=1 \cdot 25$, the distribution of $\mathrm{TI}_{w} / V$ is different in both magnitude and order than in the case of $\mathrm{TI}_{u} / V$. In Figure 17(b), very weak vertical turbulence intensity 


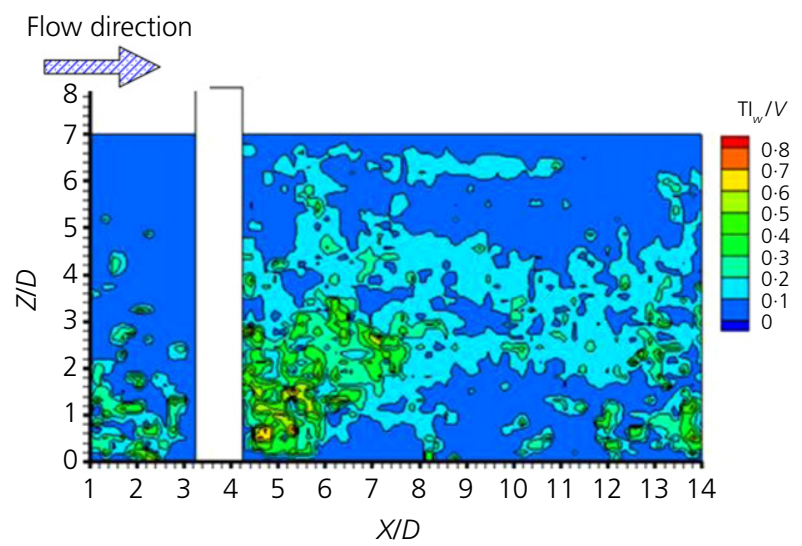

(a)

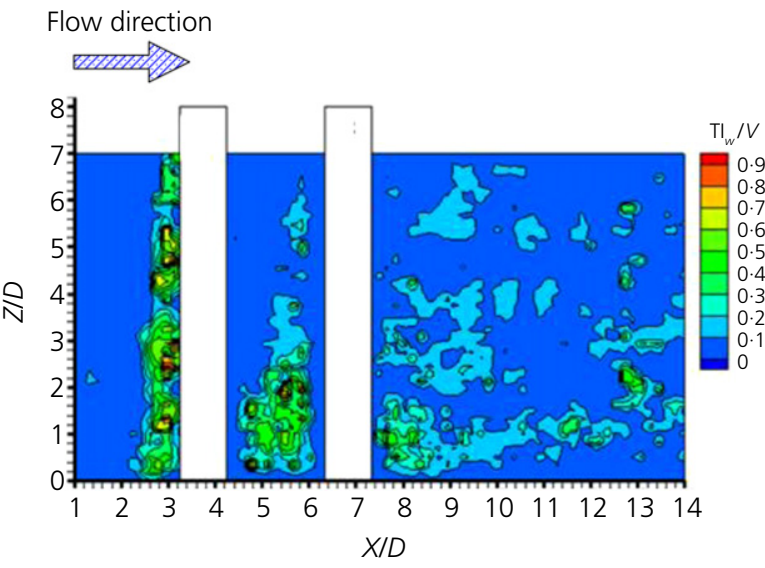

(c)

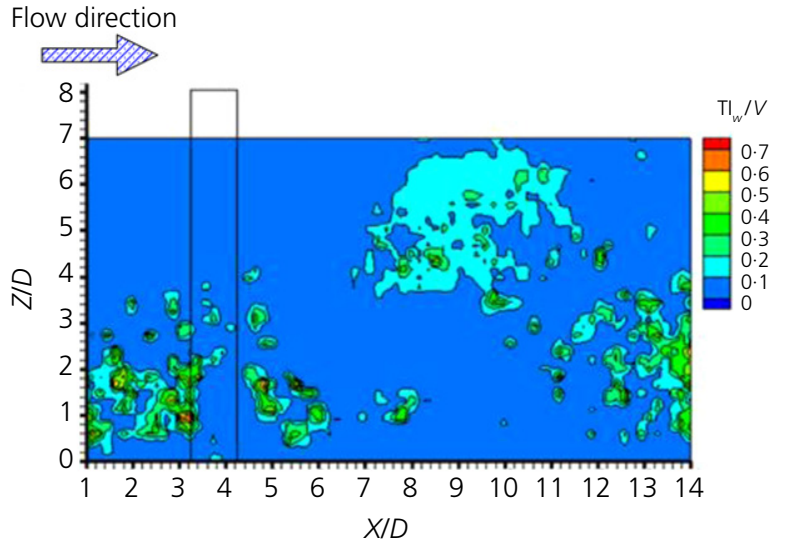

(b)
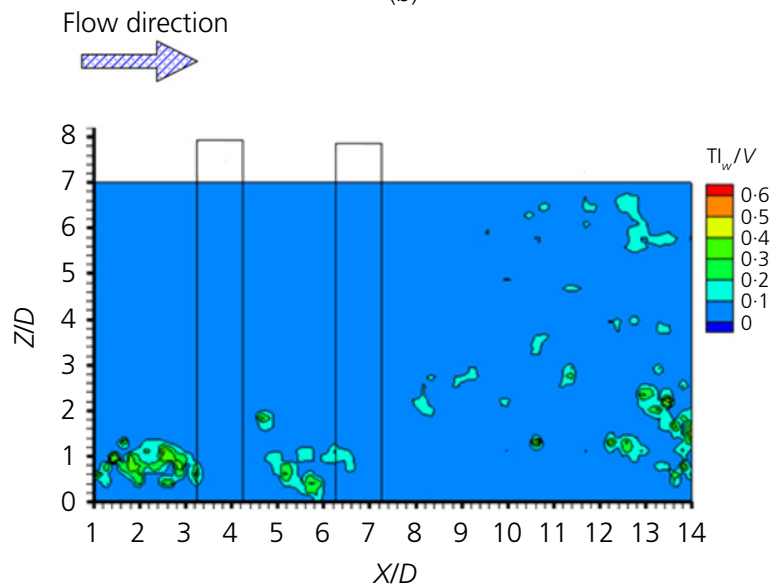

(d)

Figure 17. Contour plots of vertical turbulence intensity components for: (a) single pier at $Y / D=0$; (b) single pier at $Y / D=1 \cdot 25$; (c) two piers with $L / D=3$ at $Y / D=0$; (d) two piers with $L / D=3$ at $Y / D=1.25$

can be noticed in the plane at $Y / D=1 \cdot 25$, except close to the bed.

For the case of two in-line piers, the distribution of $\mathrm{TI}_{w} / V$ for $L / D=3$ is presented in Figures 17(c) and 17(d). For all the values of $L / D$, approximately the same order and distribution of $\mathrm{TI}_{w} / V$ is observed at the upstream side of pier 1. Similarly, for the plane $Y / D=1 \cdot 25$, the distribution of vertical turbulence intensity at the upstream side of the pier 1 for all values of $L / D$ is almost constant. Between two in-line piers, higher values of $\mathrm{TI}_{w} / V$ are observed, close to the bed. The value decreased towards the free surface as in the case of $\mathrm{TI}_{u} / V$. When the value of $L / D$ is less than or equal to 3 , the vertical turbulence intensity in the gap near the bed is observed with the maximum value in the range of $0 \cdot 4-0 \cdot 5$. For $L / D \geq 4$, however, the maximum value of $\mathrm{TI}_{w} / V$ is approximately equal to 0.4 close to pier 1 , gradually decreases as the flow approaches pier 2 and eventually reaches a condition similar to the upstream side of pier 1 . It is noticed that the maximum values of $\mathrm{TI}_{u} / V$ and $\mathrm{TI}_{w} / V$ between the two piers for all the values of $L / D$ are approximately the same.
The maximum value of $\mathrm{TI}_{w} / V$ for $L / D=1$ at the downstream side of pier 2 is about $50 \%$ less than that of the single pier case. The value of $\mathrm{TI}_{w} / V$ increases as the spacing between the two piers increases. It is also common to observe that $\mathrm{TI}_{w} / V$ gradually decreases in the downstream direction in all the cases of $L / D$. For the plane $Y / D=1 \cdot 25$, when $L / D \leq 3$, a similar distribution of $\mathrm{TI}_{w} / V$ is observed, with a value approximately equal to $0 \cdot 1$. However, for $L / D>3$ a slight increase in $\mathrm{TI}_{w} / V$ is noticed at the bank of the downstream side of pier 2 , which is further extended towards the downstream side.

\subsection{Turbulent kinetic energy}

Contour plots of TKE $/ V^{2}$ for the single pier case in the vertical planes at $Y / D=0$ and 1.25 are presented in Figures 18(a) and 18(b). The results from the contour plots indicate that the distribution of TKE/ $V^{2}$ is similar to the distribution of turbulence intensity. At the upstream side of a pier, higher values of turbulent kinetic energy are observed close to the bed and gradually decrease towards the free surface. The maximum value of $\mathrm{TKE} / V^{2}$ is approximately equal to $0 \cdot 3$ at the upstream side of a pier. At the downstream side of a pier, the maximum 


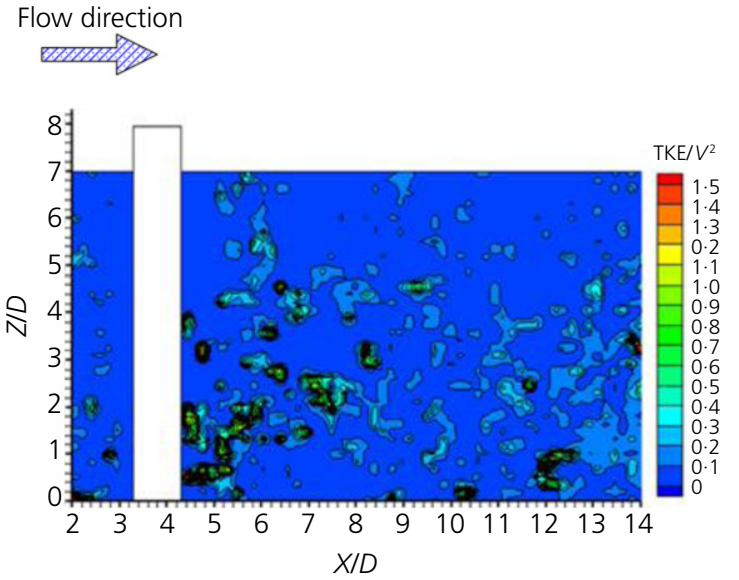

(a)

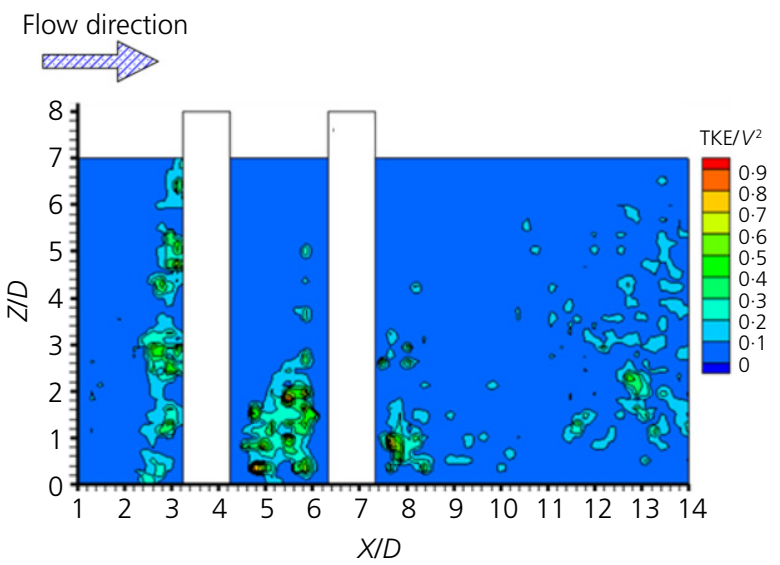

(c)

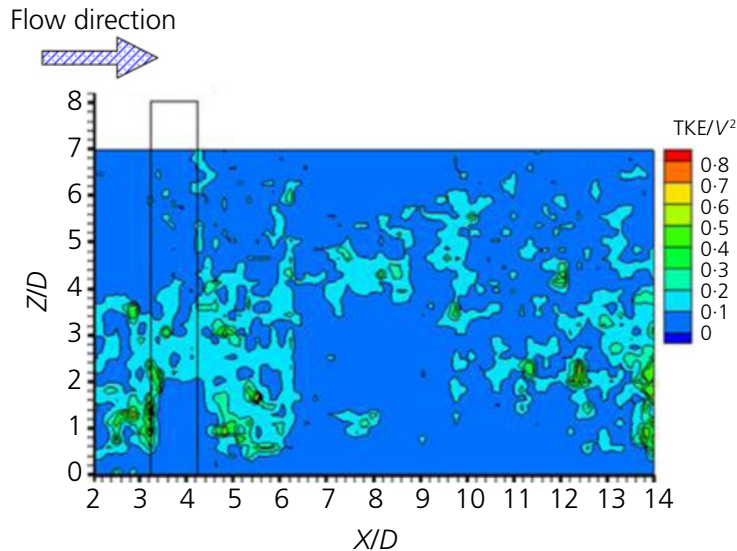

(b)
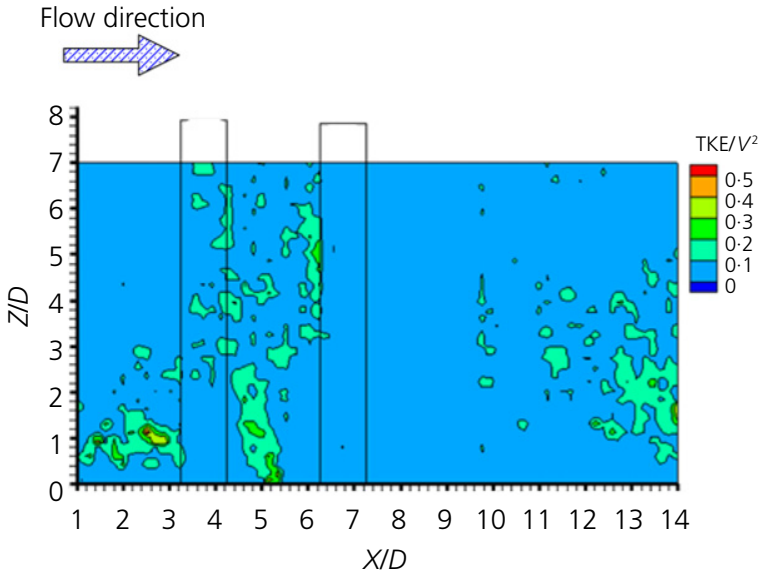

(d)

Figure 18. Contour plots of turbulent kinetic energy for: (a) single pier at $Y / D=0$; (b) single pier at $Y / D=1 \cdot 25$; (c) two piers with $L / D=3$ at $Y / D=0 ;(d)$ two piers with $L / D=3$ at $Y / D=1.25$

value of $\mathrm{TKE} / V^{2}$ is observed close to the pier near the bed. It is about two times greater than that of the upstream side. The result indicates that the value of turbulence kinetic energy decreases as the distance increases further in the downstream side direction.

For the cases of two in-line piers, contour plots of TKE/ $V^{2}$ are presented in Figures 18(c) and 18(d) for $L / D=3$. Results for various $L / D$ values indicate that there is a significant change in the turbulence kinetic energy from upstream to downstream of the piers. At the upstream side of pier 1, the distributions of TKE $/ V^{2}$ for all the cases of $L / D$ are similar to the single pier case. Between the two piers and at the downstream side of pier 2, the results show that the distribution of $\mathrm{TKE} / V^{2}$ is more or less similar to that of the turbulence intensity. An increase occurs in the turbulent kinetic energy behind pier 1 as the spacing between the two piers increases. For the case of $L / D=3$ and 4 , the pocket of higher value of turbulent kinetic energy occurs close to the pier 2. However, for $L / D>4$, the value of turbulent kinetic energy decreases as the flow approaches pier 2. For example, in the case of $L / D=6$, the value of TKE/ $V^{2}$ close to pier 2 at the upstream side is approximately equal to $0 \cdot 1$, about $70 \%$ less than that of the downstream side of pier 1 . At the downstream side of pier 2 , the value of $\mathrm{TKE} / V^{2}$ increases as the spacing between the two piers increases up to $L / D=3$. Further increase in the spacing results in a decrease in the value of $\mathrm{TKE} / V^{2}$.

\subsection{Reynolds shear stresses}

Reynolds shear stresses are calculated using only the streamwise and vertical components of velocity fluctuation and are denoted by $-\rho \overline{u^{\prime} w^{\prime}}$. The values of $-\rho \overline{u^{\prime} w^{\prime}}$ have been normalised by $\rho \# \mathrm{~V}^{2}$. Here, $\rho$ represents the mass density of water.

The contour plots of Reynolds shear stresses on two different longitudinal planes $Y / D=0$ and 1.25 for the single pier case are shown in Figures 19(a) and 19(b). The contour plots 
indicate that, at the upstream side of a pier, Reynolds shear stresses considerably increase as the flow approaches close to the pier. Small pockets of positive as well as negative values of Reynolds shear stresses are observed near the bed close to the pier. The Reynolds stress values at the upstream side are in the range from $-0 \cdot 3$ to $0 \cdot 2$. At the downstream side of a pier, a similar trend for the Reynolds shear stresses is observed, with higher values close to the bed. In the $Y / D=0$ plane, a pocket of positive and negative values in the range from -0.5 to 0.5 is observed close to the pier and extends up to a distance of $3 D$ downstream, measured from the face of the pier. However, in the plane $Y / D=1 \cdot 25$, a positive value of Reynolds shear stress approximately equal to $0 \cdot 3$ is observed throughout the depth.

For the cases of two in-line piers, the contour plots of Reynolds shear stress for $L / D=3$ are presented in Figures 19(c) and 19(d). The results at the upstream side of pier 1 for all values of $L / D$ indicate similar trends of Reynolds shear stress distribution. However, at the downstream side of pier 2 and between the two piers, a significant increase in the values of Reynolds shear stresses was observed. Between the two piers, it

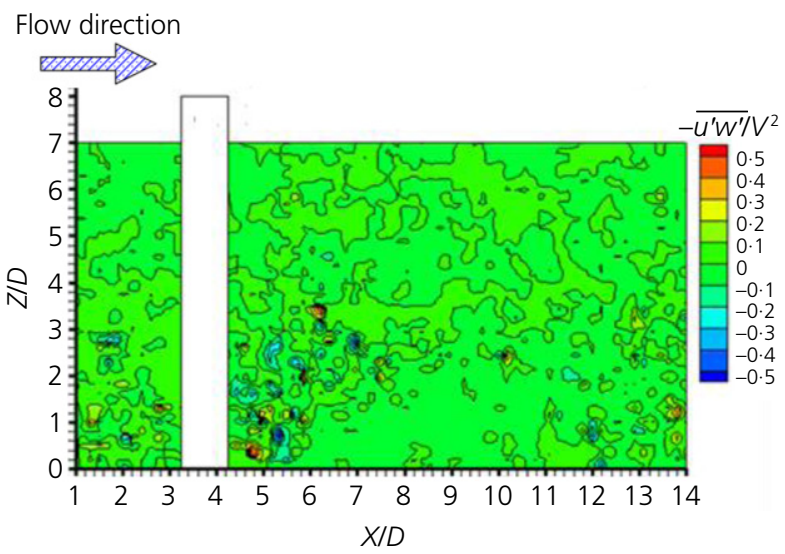

(a)

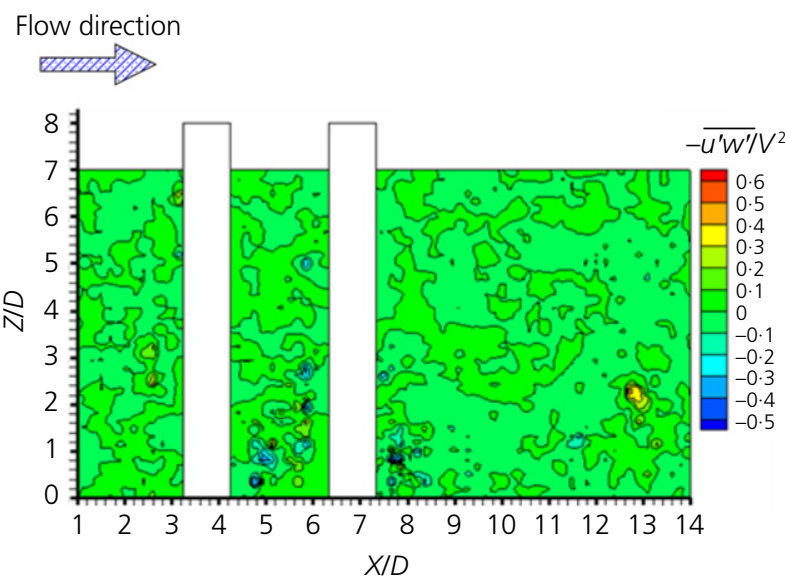

(c) is common to observe the higher negative values close to the bed. Moreover, it is observed that the value of this parameter decreases with increase in the spacing between the two in-line piers. Higher values are observed close to pier 1 and gradually decrease as the flow approaches pier 2. For example, in the case of $L / D=6$, Reynolds shear stress is in the range from $-0 \cdot 1$ to $0 \cdot 1$ close to pier 1 , whereas it is approximately equal to zero close to pier 2 . In the wake of pier 2 , an increase in the value is noticed with increase in the distance between the two piers until $L / D=3$. Further increase in the value of $L / D$ decreases Reynolds shear stress at the wake of pier 2. For $L / D \leq 3$, the maximum value is approximately equal to $0 \cdot 2$ at the wake of pier 2 . For $L / D>3$, an approximate range from $-0 \cdot 1$ to $0 \cdot 1$ is observed.

The results of this study have been summarised at Table 2 for the longitudinal plane at $Y / D=0$.

\section{Conclusions}

The PIV technique was used to study flow structures and turbulence characteristics around a single pier and two in-line

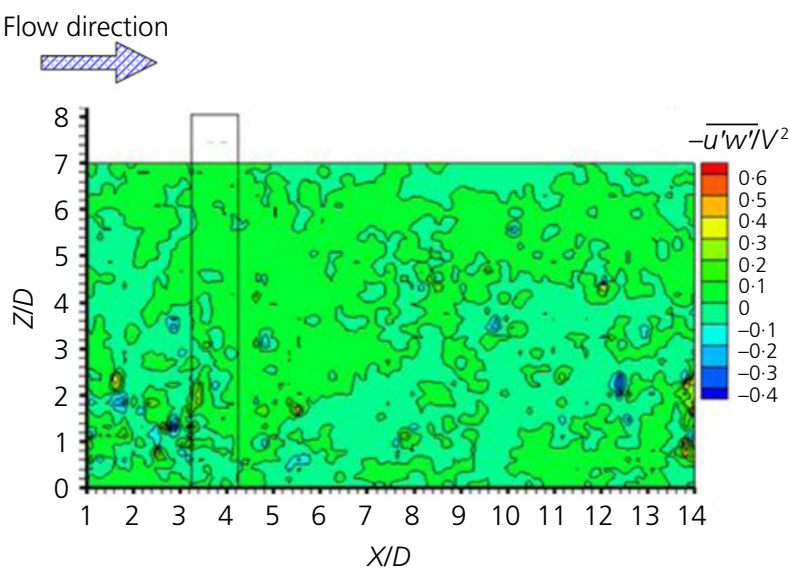

(b)

\section{Flow direction} mowe

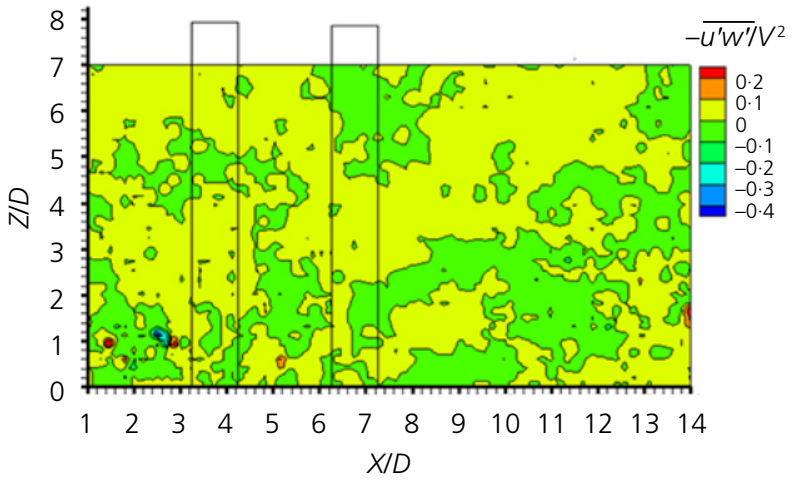

(d)

Figure 19. Contour plots of Reynolds shear stress for: (a) single pier at $Y / D=0$; (b) single pier at $Y / D=1 \cdot 25$; (c) two piers with $L / D=3$ at $Y / D=0 ;(d)$ two piers with $L / D=3$ at $Y / D=1 \cdot 25$ 


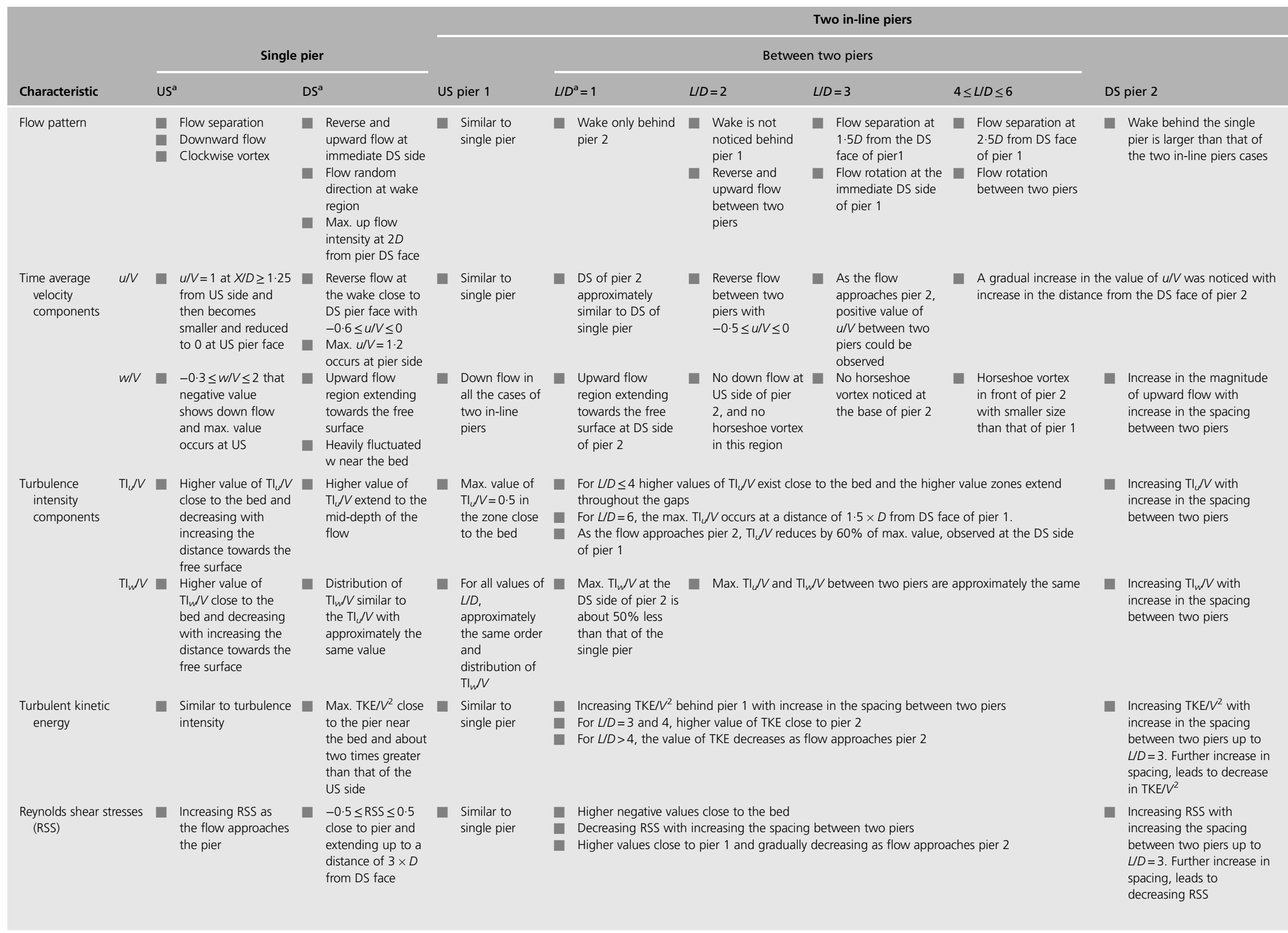


cylindrical bridge piers. The results demonstrate that the flow structures around the two in-line piers are more complicated than that of the single pier case. Furthermore, the spacing between two in-line piers significantly affect the flow structures and particularly the wake of the piers. The turbulence characteristics such as turbulence intensity, turbulent kinetic energy and Reynolds shear stresses are notably different from those for the single pier. Results of this study show that, when the spacing is in the range of $2 \leq L / D \leq 3$, stronger turbulence structures are noticed behind pier 1 and, as a result, a higher scour depth can be expected around pier 1. Accordingly, it can be concluded that, for the tandem arrangements of two in-line cylindrical piers, spacing in the range of $2 \leq L / D \leq 3$ is the most critical at which the piers experience the highest turbulence, and hence the maximum scour depth can be expected. The experiments of this study were conducted in a fixed bed flume with no sediment layer. In a companion set of experiments conducted by the present authors, the flow structure and corresponding local scour were measured at different pier spacing in a flume with a sediment layer. The results will be published in a follow-up paper.

\section{REFERENCES}

Ahmed F and Rajaratanam N (1997) The three dimensional turbulent boundary layer flow around bridge piers. Journal of Hydraulic Research 35(2): 209-224.

Chiew YM (1984) Local Scour at Bridge Piers. PhD thesis, Department of Civil Engineering, Auckland University, Auckland, New Zealand.

Dargahi B (1989) The turbulent flow field around a circular cylinder. Experiments in Fluids 8(1): 1-12.

Elhimer M, Harran G, Hoarau Y et al. (2016) Coherent and turbulent processes in the bistable regime around a tandem of cylinders including reattached flow dynamics by means of high-speed PIV. Journal of Fluids and Structures 60(1): 62-79.

Ettema R (1980) Scour at Bridge Piers. PhD thesis, Department of Civil Engineering, The University of Auckland, Auckland, New Zealand.

Ettema R, Constantinescu G and Melville B (2011) Evaluation of Bridge Scour Research: Pier Scour Process and Predictions. Transportation Research Board of the National Academies, Washington, DC, USA, NCHRP Report 175.

Greifzu F, Kratzsch C, Forgber T, Lindner F and Schwarze R (2016) Assessment of particle-tracking models for dispersed particle-laden flows implemented in OpenFOAM and ANSYS FLUENT. Engineering Applications of Computational Fluid Mechanics 10(1): $30-43$.

Hamill L (1999) Bridge Hydraulics. Routledge, New York, NY, USA. Igarashi T (1981) Characteristics of the flow around two circular cylinders arranged in tandem: 1st report. Bulletin of JSME 24(188): 323-331.

Kumar A and Kothyari U (2012) Three-dimensional flow characteristics within the scour hole around circular uniform and compound piers. Journal of Hydraulic Engineering 138(5): 420-429.

Melville BW (1975) Local Scour at Bridge Sites. School of Engineering, University of Auckland, Auckland, New Zealand.

Melville BW and Coleman SE (2000) Bridge Scour. Water Resource Publications, LLC, Littleton, CO, USA.

Melville BW and Raudkivi AJ (1977) Flow characteristics in local scour at bridge piers. Journal of Hydraulic Research 15(4): 373-380.
Meneghini J, Saltara F, Siqueira C and Ferrari J Jr (2001) Numerical simulation of flow interference between two circular cylinders in tandem and side-by-side arrangements. Journal of Fluids and Structures 15(2): 327-350.

Mohammadpour R, Ghani AA, Zakaria NA and Ali TAM (2015) Predicting scour at river bridge abutments over time. Proceedings of the Institution of Civil Engineers - Water Management 170(1): 15-30, http://dx.doi.org/10.1680/jwama.14.00136.

Muzzammil M and Gangadhariah T (2003) The mean characteristics of horseshoe vortex at a cylindrical pier. Journal of Hydraulic Research 41(3): 285-297.

Okajima A, Yasui S, Kiwata T and Kimura S (2007) Flow-induced streamwise oscillation of two circular cylinders in tandem arrangement. International Journal of Heat and Fluid Flow 28(4): $552-560$.

Özkan F, Wenka A, Hansjosten E, Pfeifer P and Kraushaar-Czarnetzki B (2016) Numerical investigation of interfacial mass transfer in two phase flows using the VOF method. Engineering Applications of Computational Fluid Mechanics 10(1): 100-110.

Qadar A (1981) The vortex scour mechanism at bridge piers. Proceedings of the Institution of Civil Engineers 71(3): 739-757, http://dx.doi.org/10.1680/iicep.1981.1816.

Richardson EV and Davis SR (2001) Evaluating Scour at Bridges, 4th edn. Federal Highway Administration, Washington, DC, USA, FHWA NHI 01-001, HEC-18.

Richardson JE and Panchang VG (1998) Three-dimensional simulation of scour-inducing flow at bridge piers. Journal of Hydraulic Engineering 124(5): 530-540.

Sheppard DM (2004) Overlooked local sediment scour mechanism. Transportation Research Record 1890: 107-111.

Shrestha CK, Keshavarzi A, Khabbaz H and Ball J (2012) Experimental study of the flow structure interactions between bridge piers. Proceedings of the 34th Hydrology and Water Resources Symposium (HWRS 2012), Sydney, Australia, pp. 308-315.

Shrestha CK, Keshavarzi A and Khabbaz H (2013) Flow structure at downstream side of two sequential bridge piers. In Proceedings of International Symposium on River Sedimentation (ISRS 2013), Kyoto, Japan (Fukuoka S, Nakagawa H, Sumi T and Zhang H (eds)). CRC Press/Balkema, Rotterdam, the Netherlands, p. 199.

Sumner D (2010) Two circular cylinders in cross-flow: a review. Journal of Fluids and Structures 26(6): 849-899.

VidPIV (2004) VidPIV Version 4.6. 2004. Computer Program. Oxford Lasers, Didcot, UK.

\section{How can you contribute?}

To discuss this paper, please email up to 500 words to the editor at journals@ice.org.uk. Your contribution will be forwarded to the author(s) for a reply and, if considered appropriate by the editorial board, it will be published as discussion in a future issue of the journal.

Proceedings journals rely entirely on contributions from the civil engineering profession (and allied disciplines). Information about how to submit your paper online is available at www.icevirtuallibrary.com/page/authors, where you will also find detailed author guidelines. 